\title{
1 Cryo-electron tomography of ASC signalling sites in pyroptotic cells
}

$11{ }^{4}$ Department of Veterinary Medicine, University of Cambridge, Madingley Road, Cambridge

12 CB3 0ES, United Kingdom

$13{ }^{5} \mathrm{MRC}$ Laboratory of Molecular Biology, Francis Crick Avenue, Cambridge, CB2 0QH, United

14 Kingdom

15 Current addresses: Institute of Biochemistry and Molecular Medicine, University of Bern,

16 Bühlstrasse 28, 3012 Bern, Switzerland (A.C.B.); Wren Therapeutics, Clarendon House,

17 Clarendon Road, Cambridge CB2 8FH, UK (L.J.H.).

$18{ }^{*}$ Corresponding authors: C.E.B., ceb27@cam.ac.uk; Y.M., ymodis@mrc-Imb.cam.ac.uk 


\section{Abstract}

20 Inflammasomes induce cell death in response to infection, chemical entities and cell damage.

21 Human genetics and animal models have identified the NLRP3 inflammasome as a gatekeeper

22 of caspase-1-dependent pyroptosis. NLRP3 activation induces polymerization of ASC into a

23 single, micron-scale perinuclear punctum, where caspase-1 is activated. These puncta have yet

24 to be imaged at sufficient resolution to resolve their ultrastructure. Here, we apply correlative

25 cryo-light microscopy and cryo-electron tomography to visualize ASC/caspase-1 puncta and

26 mitochondrial membrane remodelling within NLRP3-activated cells. The puncta are composed

27 primarily of branched ASC filaments. The N-terminal pyrin domain forms an 8-10 nm-diameter

28 tubular core, which is decorated by the flexibly linked C-terminal caspase recruitment domain.

29 The variable filament density allows ribosomes and trans-Golgi-like vesicles to permeate the

30 network. We propose this provides structural integrity while allowing macromolecules and

31 vesicles to diffuse in or bind with the necessary density, timing and localization.

\section{Keywords}

33 NLR family pyrin domain containing 3 (NLRP3); apoptosis-associated speck-like protein

34 containing a CARD (ASC) speck; caspase-1; pyroptosis; correlative light and electron

35 microscopy (CLEM); In situ cryo-electron tomography (cryo-ET); focused-ion beam (FIB) milling;

36 inflammasome; caspase activation and recruitment domain (CARD); pyrin domain (PYD)

37 Running title: Cryo-ET of ASC specks and mitochondria in cell lamellae 


\section{Introduction}

41 Inflammasomes are vital innate immune oligomeric signalling complexes that induce proteolytic

42 activation of caspase-1, leading to secretion of proinflammatory cytokines interleukin (IL)-1 $\beta$ and

43 IL-18, and cell death by gasdermin D-driven pyroptosis. In the first step of inflammasome

44 assembly, Nod-like receptors (NLR) sense cytosolic chemical signatures associated with

45 microbes and cell dysfunction or damage ${ }^{1}$. Ligand binding induces NLR oligomerization and

46 recruitment of downstream proteins linked to the caspase-1 signalling cascade.

NLRP3 is one of the most studied inflammasome NLRs due to the large number of

NLRP3 genetic variants associated with human diseases and the availability of animal disease

models. At least 250 human NLRP3 protein variants have been identified, over 30 of which have been associated with disease through gain-of-function ${ }^{2,3}$, including autoinflammatory diseases, degenerative disorders, dermatitis and metabolic disorders ${ }^{4,5}$. NLRP3 inflammasome activation has been linked to a broad spectrum of cellular processes or cytosolic chemical signatures

53 including potassium efflux, reactive oxygen species, calcium influx, chloride efflux,

54 phosphatidylinositol-4-phosphate on Golgi membranes, and mitochondrial stress or damage ${ }^{6,7}$.

55 Cryo-electron microscopy (cryo-EM) single-particle image reconstructions show that ligand-

56 bound inflammasome NLRs assemble into disk- or split washer-like oligomers ${ }^{8-11}$. In addition to

57 ligand recognition, activation of the NLRP3 and pyrin inflammasomes requires active transport

58 along microtubules by dynein to the microtubule organizing centre (MTOC) $)^{12,13}$. Upon activation,

59 NLRP3 recruits the adaptor protein ASC (Apoptosis-associated speck-like protein containing a

60 caspase recruitment domain (CARD)). NLRP3 and ASC interact through their pyrin domains

61 (PYDs) ${ }^{14}$. ASC then assembles into a single perinuclear punctum with a micron-scale diameter,

62 also known as a speck, which functions as a platform for the recruitment and activation of

63 caspase $-1^{15,16}$. The PYD and CARD of ASC are both required for punctum formation and

64 signalling ${ }^{15,17,18}$. Structural studies on purified ASC PYD and ASC CARD show that both 
65 domains have a death fold and independently form helical filaments ${ }^{19-24}$. In the cell, fluorescence

66 microscopy imaging suggests that PYD-PYD interactions drive ASC filament formation whereas

67 CARD-CARD interactions promote cross-linking and compaction of PYD filaments into

68 puncta $^{17,18,23-26}$. In addition, ASC recruits procaspase-1 via a CARD-CARD interaction between

69 the two proteins ${ }^{15,23}$. Super-resolution light microscopy of endogenous ASC specks revealed

70 punctate structures containing ASC, caspase 1 and NLRP3 within the same macro-assembly ${ }^{27}$.

71 These structures have yet to be imaged inside a cell at sufficient resolution to resolve the ASC

72 or caspase-1 ultrastructure within puncta ${ }^{26}$. Hence, it remains unclear how the PYD and CARD

73 contribute to the formation of an ASC punctum inside cells.

Understanding how ASC filament formation contributes to the assembly and activation of

75 inflammasome puncta requires structural information in the cellular context. Recent advances in

76 the preparation of frozen vitrified cellular lamellae by focused ion-beam (FIB) milling ${ }^{28,29}$ have

77 allowed the ultrastructure of multimeric protein complexes to be determined in a near-native

78 context by cryo-electron tomography (cryo-ET). Here, we use in situ cryo-ET to obtain three-

79 dimensional image reconstructions of multimeric signalling complexes in cells with active

80 NLRP3 inflammasomes. We identify ASC/caspase-1 signalosome puncta by correlative light

81 and electron microscopy (CLEM) and reveal their ultrastructure and pyroptotic cellular

82 landscape in immortalised bone marrow derived macrophages (iBMDMs) by cryo-ET image

83 reconstruction. We find that inflammasome puncta are composed of short, hollow ASC/caspase-

841 filaments, extensively branched, with variable packing density allowing ribosomes and certain

85 other cellular components to permeate the puncta. We propose that this ultrastructure of the

86 ASC filament network functions as a signalling platform by providing structural integrity while

87 allowing downstream signalling molecules to diffuse freely and bind at high density within the

88 network. 


\section{Results}

\section{In situ cryo-CLEM of ASC/caspase-1 puncta}

91 High-resolution structures of inflammasome components have been obtained from purified

92 proteins. In a previous study in zebrafish larvae, electron tomography of ASC puncta in fixed

93 larval sections stained with uranyl acetate showed that the puncta contained a dense filament

94 network ${ }^{26}$, but the imaging resolution was limited by chemical fixation and staining of the

95 sample. To examine the architecture and cellular interactions of the mammalian NLRP3

96 signalosome in its physiological environment, we imaged ASC puncta in iBMDMs by correlative

97 fluorescence light microscopy and in situ cryo-ET (cryo-CLEM). Cells expressing ASC fused

98 with a C-terminal fluorescent protein have been used extensively to visualize ASC puncta

99 formation ${ }^{30}$. In some experimental systems, ASC overexpression can lead to puncta formation

100 in the absence of activating inflammasome stimuli ${ }^{30}$. Fluorescence microscopy of live iBMDM

101 cells overexpressing ASC-mCerulean used in this study showed that both priming with

102 lipopolysaccharide (LPS) and stimulation with nigericin were still required to induce ASC puncta

103 formation (Fig. S1a, Movie S1). Gasdermin D cleavage observed by Western blotting and IL-1 $\beta$

104 cytokine release measured in ELISAs confirmed that the ASC-mCerulean puncta activated

105 pyroptotic signalling (Fig. S1b). Limiting the stimulation time with nigericin to 30 min allowed

106 early-stage inflammasome signalling complexes to be captured, as indicated by colocalization of

107 NLRP3, proteolytically activated caspase-1 and IL-1 $\beta$ with ASC in the puncta, in both wild-type

108 (WT) iBMDMs and ASC-mCerulean iBMDMs (Fig. S1c-e). NLRP3 and IL-1 $\beta$ localization was

109 determined by immunofluorescence. Caspase-1 localization was inferred using the

110 carboxyfluorescein-labelled caspase-1 substrate FAM-FLICA. FAM-FLICA or its non-fluorescent

111 analogue Z-VAD-FMK also facilitated imaging by delaying pyroptotic cell death (Movies S1, 112 S2). 
For high-resolution imaging, cells grown on electron microscopy grids were vitrified by

114 plunge-freezing in liquid ethane. We used both iBMDMs overexpressing ASC-mCerulean and

115 WT iBMDMs labelled with FAM-FLICA after NLRP3 inflammasome activation ${ }^{31}$. ASC/caspase-1

116 signalling complexes were located by cryo-fluorescence light microscopy (cryo-FM; Fig. S2a).

117 Areas of the frozen cells containing an ASC or caspase punctum were FIB-milled down to

118 lamellae $150-300 \mathrm{~nm}$-thick and 15-20 $\mu \mathrm{m}$ wide ${ }^{28,29}$, with micro-expansion joints to reduce

119 bending ${ }^{32}$ (Fig. S2b,c). The lamellae were imaged by cryo-CLEM, using cryo-FM to locate any

120 remaining fluorescent signal from ASC-mCerulean or FAM-FLICA in the lamellae after milling

121 (Fig. 1a,b; Fig. S2d,e). The micron-scale dimensions of the fluorescent puncta allowed cryo-ET

122 tilt-series acquisition at regions of interest with the necessary targeting precision after

123 correlation of the cryo-FM images with low-magnification cryo-EM maps (Fig. S2f,g) ${ }^{33,34}$.

124 In situ Cryo-ET reveals a branched-filament ultrastructure in ASC/caspase-1 puncta

125 Three-dimensional cryo-ET image reconstructions show a dense network of branched filaments

126 in areas identified by cryo-CLEM as containing ASC-mCerulean or caspase-1 labelled with

127 FAM-FLICA (Figs. 1c-e, 2a, S2g). Filaments in cells expressing ASC-mCerulean had additional

128 density at the filament periphery, and a less distinct outline compared to filaments in cells

129 expressing WT ASC (Fig. 1d,e). This suggests the additional density is primarily attributable to

130 mCerulean, which at $27 \mathrm{kDa}$ is larger than ASC (22 kDa), although other components such as

131 caspase-1 (which was shown by light microscopy to localize to ASC specks), may also

132 contribute to this density. A green fluorescent protein tag was similarly found to decorate cryo-

133 ET reconstructions of amyloid-like poly-Gly-Ala aggregates with additional densities ${ }^{35}$. Since

134 mCerulean was fused to the C-terminus of ASC, the presence of mCerulean at the filament

135 periphery supports a model in which the filaments are formed at their core by the $\mathrm{N}$-terminal

136 PYD of ASC, with the CARD located in between the PYD and mCerulean. Indeed, the cryo-EM

137 structure of purified recombinant ASC PYD filaments and the NMR structure of full-length ASC, 
138 taken together, suggest that the PYD forms the filament core (9-nm in diameter), with the

139 flexibly-linked CARD increasing the filament diameter to $16-18 \mathrm{~nm}^{23,24}$. In our cryo-ET

140 reconstructions, filaments containing WT ASC had a diameter of $11-15 \mathrm{~nm}$, which increased to

141 22-30 nm for filaments containing ASC-mCerulean (Figs. 1d,e). Notably, a continuous core of

142 density 8-10 $\mathrm{nm}$ in diameter (Fig. 2a-c) was visible within the ASC-mCerulean filaments (for

143 which the cryo-ET images were of higher quality). Although fluorescence from ASC-mCerulean

144 and FAM-FLICA-labelled capsase-1 colocalized in iBMDMs (Fig. S1e), the resolution of cryo-

145 CLEM was insufficient to determine whether any of the cryo-ET filament densities corresponded

146 to caspase-1 or other components. We note, however, that caspase-1 (30 kDa) and

147 procaspase-1 (45 kDa) are both larger than ASC and would therefore be expected to increase

148 filament diameter significantly, if present with the same stoichiometry as ASC.

149 Filament length measurements showed that WT filaments were on average slightly

150 shorter $(191 \pm 74 \mathrm{~nm}, \mathrm{n}=54)$ than ASC-mCerulean filaments $(245 \pm 116 \mathrm{~nm}, \mathrm{n}=402)$, with

151 similarly broad length distributions (Fig. 1f,g). This difference could potentially be due to the

152 higher levels of ASC protein expression in iBMDMs expressing ASC-mCerulean, which also

153 express endogenous WT ASC. Analysis of the branching angles showed that the angle

154 distributions of untagged and tagged filaments were slightly different, with larger branching

155 angles more common in mCerulean-tagged filaments, potentially due to steric constraints from

156 the tag (Fig. 1h). Our filament branching angle measurements are likely underestimated as any

157 filaments with their axis closely aligned with the Z-axis - which would result in a large $\left(70-90^{\circ}\right)$

158 branching angle - would not have been detected due to the missing-wedge effect (from sample-

159 tilt limitations). Taken together with previously published structural data from purified proteins,

160 our cryo-ET reconstructions suggest ASC filaments form the backbone of inflammasome-

161 induced puncta, but that the branching of these filaments results in a structural scaffold that can 162 recruit and concentrate downstream effector proteins. 

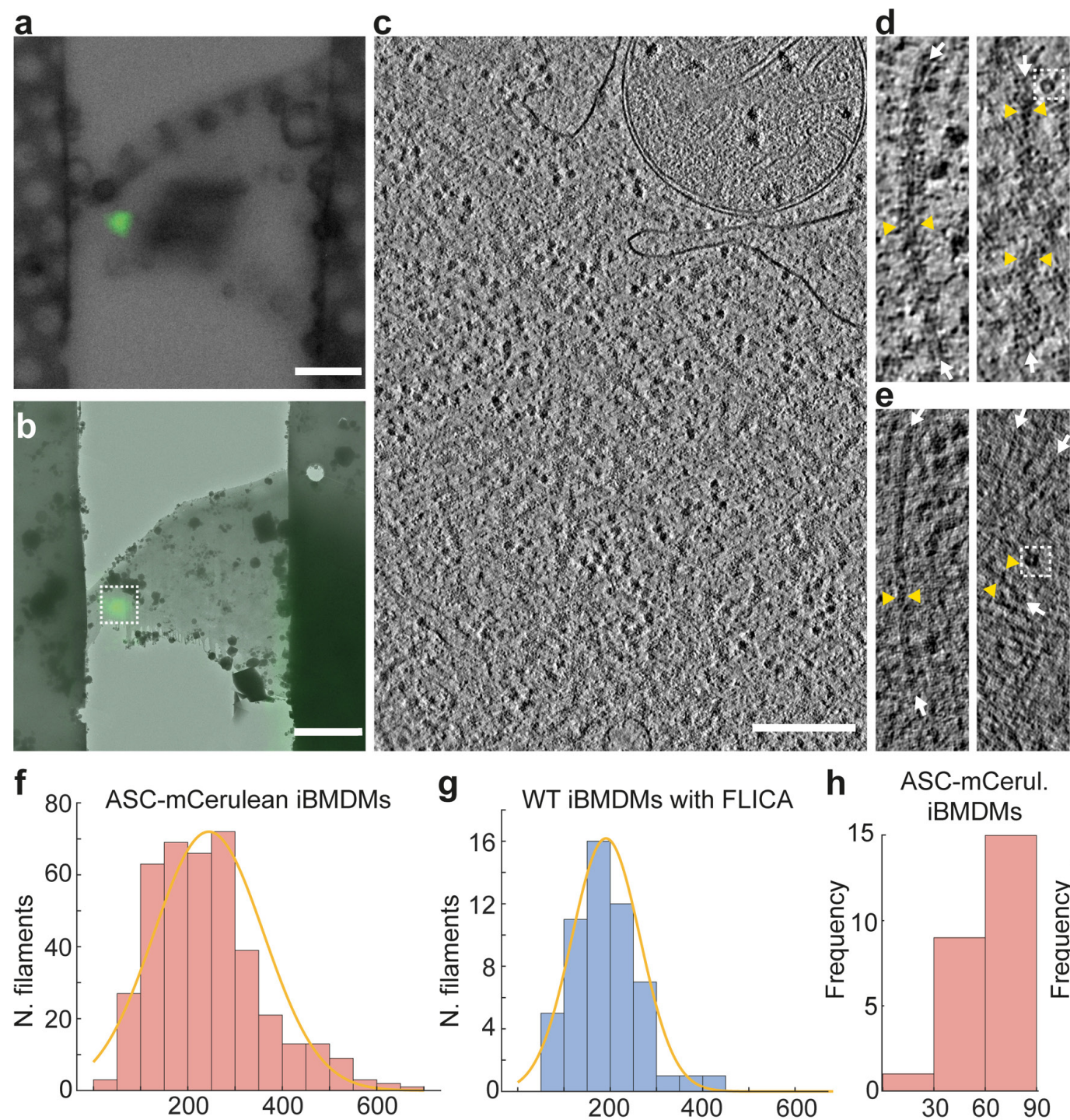

163

Filament length $(\mathrm{nm})$

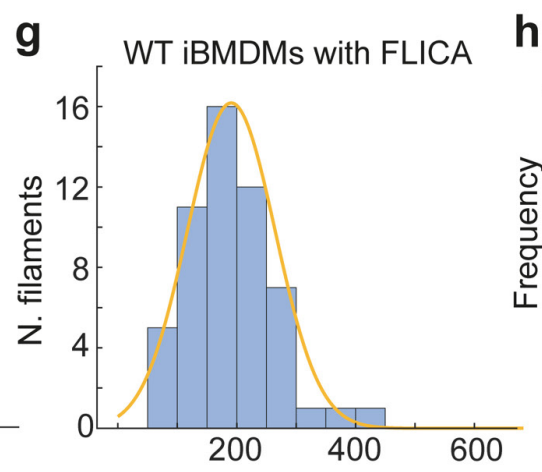

Filament length $(\mathrm{nm})$ h ASC-mCerul. iBMDMs
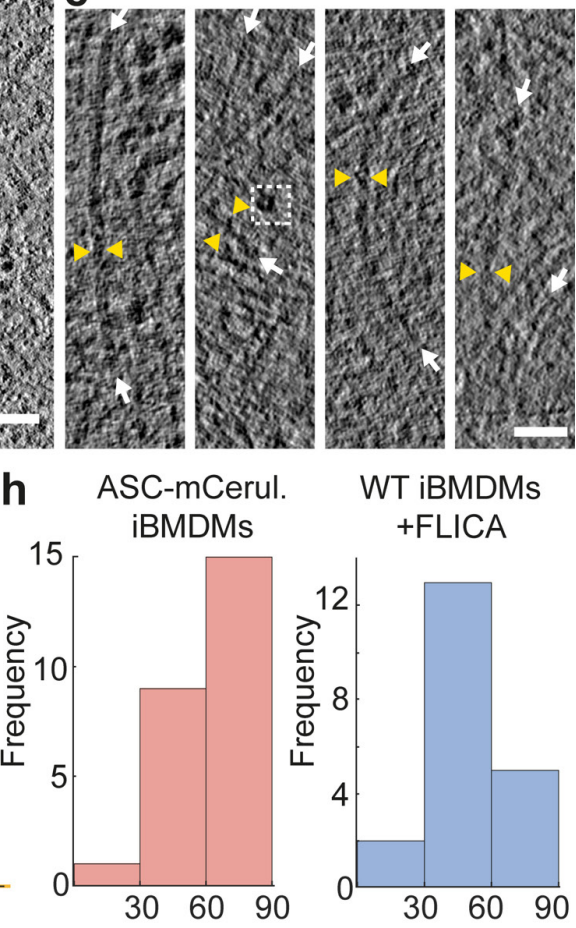

WT iBMDMs + FLICA

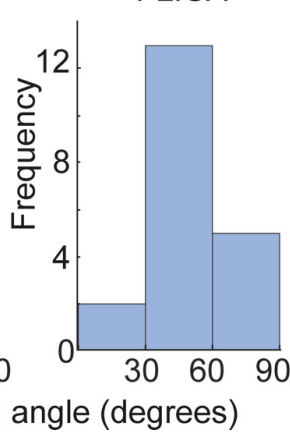

164 Figure 1. Cryo-ET image reconstructions of ASC-caspase 1 puncta in iBMDMs.

165 (a) Cryo-FM image of a lamella from an iBMDM expressing ASC-mCerulean. ASC-mCerulean

166 fluorescence is shown (green), overlaid on a bright-field image. Scale bar, $5 \mu \mathrm{m}$.

167 (b) Cryo-FM image shown in (a) correlated and overlaid on a transmission cryo-EM map of the 168 same lamella using the EC-CLEM ICY ${ }^{36}$ plugin. Scale bar, $8 \mu \mathrm{m}$.

169 (c) Reconstructed cryo-ET volume (1.36-nm thick virtual tomographic slice), of the ASC-

170 mCerulean punctum shown in $(\mathbf{a}, \mathbf{b})$. Scale bar, $250 \mathrm{~nm}$.

171 (d-e) Galleries of filaments within ASC-mCerulean puncta, (d), or FAM-FLICA-labelled caspase1721 puncta, (e), in 4-nm thick virtual tomographic slices of iBMDMs. White arrows indicate 
173 filament axes. Pairs of yellow triangles show filament thickness. Dashed white boxes 174 highlight ring-shaped densities. Scale bars, $50 \mathrm{~nm}$.

175 (f-g) Filament length distribution in iBMDMs expressing ASC-mCerulean, (f), and in WT 176 iBMDMs labelled with FAM-FLICA, (g).

177 (h) Distribution of branching angles in ASC-mCerulean puncta (from 2 tomograms), and a $178 \quad$ WT ASC punctum (1 tomogram).

For a more complete visualisation of the ASC speck ultrastructure in its cellular

180 environment, we generated a three-dimensional model of the filament network and other cellular 181 components including mitochondria, ER and ribosomes with IMOD ${ }^{37}$. This model showed that

182 puncta consist of several core regions of densely packed filaments separated by sparser

183 regions, with branching filaments connecting adjacent densely packed regions (Fig. 2a, Movies

184 S3,S4). Ribosomes were abundant within the filament network. Vesicles were also present, but 185 organelles including mitochondria were excluded. We next investigated the equilibrium dynamics of ASC-mCerulean filaments by

187 fluorescence recovery after photobleaching (FRAP) in iBMDMs. We bleached circular regions 188 containing ASC-mCerulean puncta and imaged the ASC-mCerulean fluorescence intensity 189 before and after bleaching (Fig. 2d). Our data showed that ASC-mCerulean filament assemblies 190 have a small mobile fraction (6.9\%; Fig. 2e). The dissociation rate $\left(k_{\text {off }}\right)$ of ASC-mCerulean from 191 the imaged area was measured from the rate of fluorescence recovery as $0.023 \pm 0.001 \mathrm{~s}^{-1}$

192 (Fig. 2d,e; Dataset S1). Most of the observed fluorescence recovery was at the periphery of 193 puncta, suggesting that the ASC-mCerulean filaments inside the puncta are largely immobile 194 and are not in equilibrium with ASC monomers or oligomers in the cytosol (Fig. 2d). 


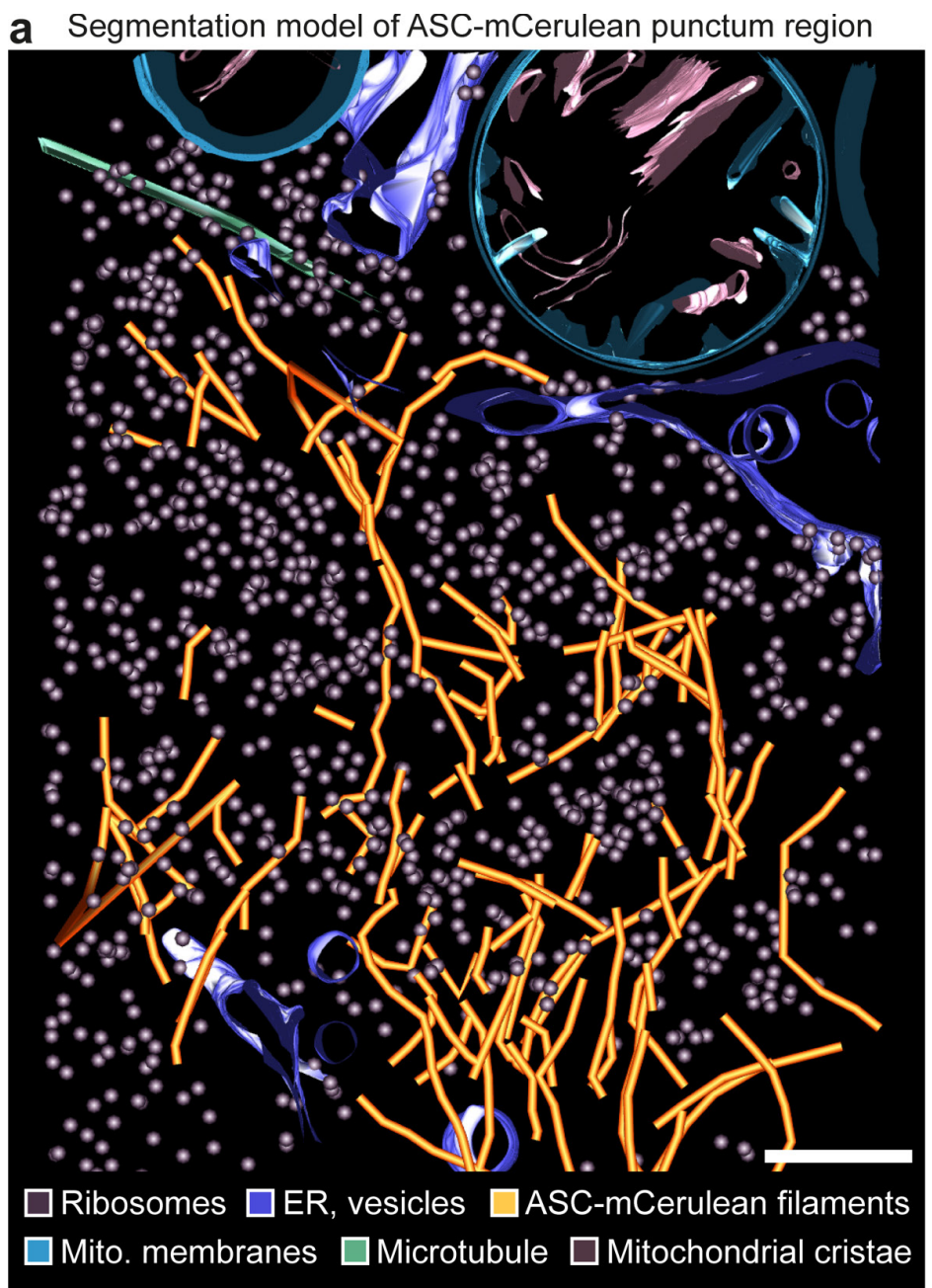

d Photobleaching of ASC-mCerulean fluorescence

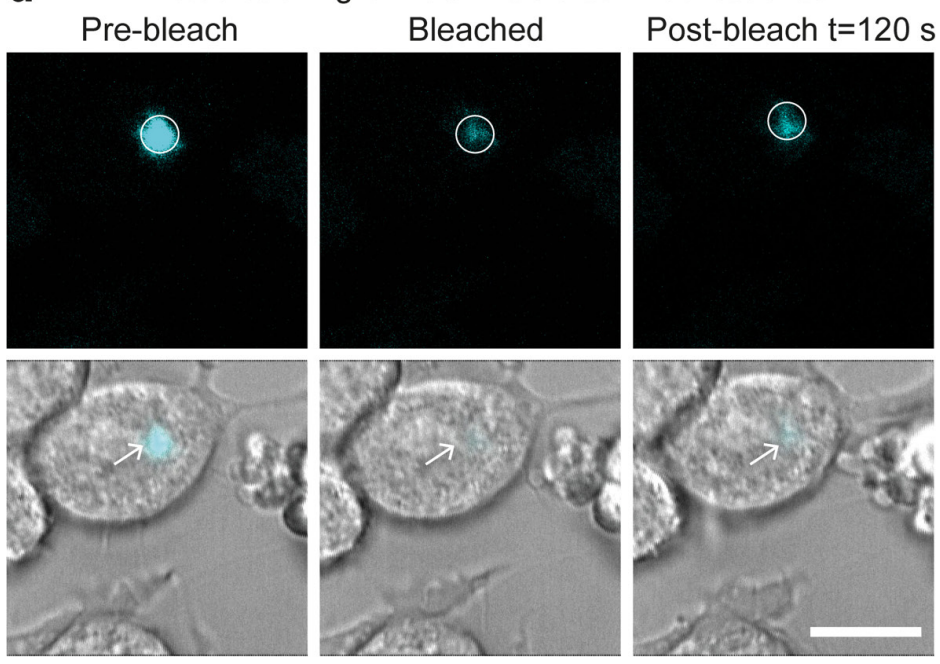

b

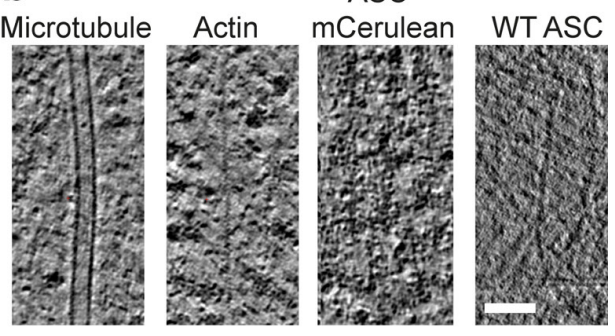

C Core filament crossection density
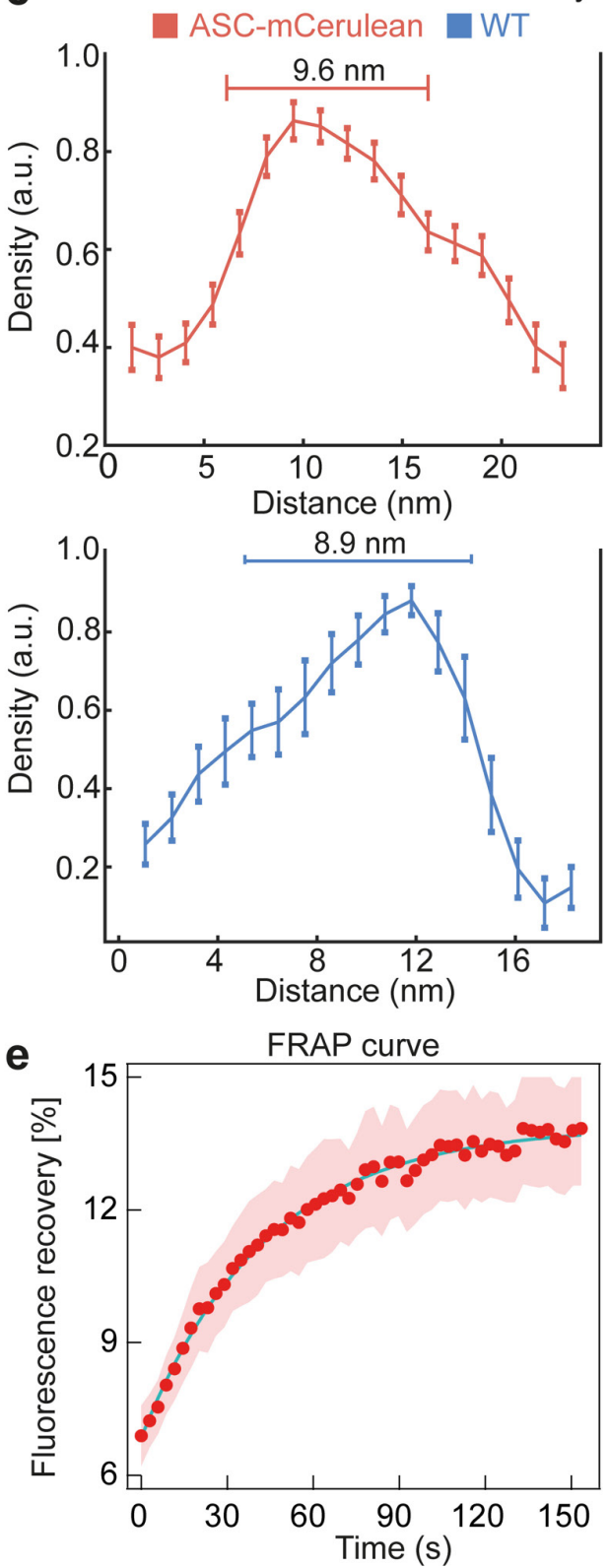

Figure 2. Visualization of the filament network and assembly dynamics of ASC puncta

197 (a) 3-D segmented model of the ASC-mCerulean punctum shown in Fig. 1c, generated with

$198 \quad$ IMOD $^{37}$. Scale bar, $250 \mathrm{~nm}$. 
199 (b) Representative instances of different filament types observed in 4-nm thick virtual

200 tomographic slices of iBMDMs. Scale bar, $50 \mathrm{~nm}$.

201 (c) Average normalized density line profile of filament cross-sections, calculated from

202 projections of 16-nm thick virtual tomographic slices of iBMDMs. Error bars show standard 203 deviations (ASC-mCerulean, $\mathrm{n}=25$; WT, $\mathrm{n}=13$ ).

204 (d) Fluorescence recovery after photobleaching (FRAP) of ASC-mCerulean in iBMDMs. Scale $205 \quad$ bar, $10 \mu \mathrm{m}$.

206 (e) FRAP curve used to calculate the ASC-mCerulean dissociation rate $\left(k_{\text {off }}\right)$ from the bleached 207 area. Shaded area represents \pm s.e.m. $(n=8)$. See Dataset S1 for source data.

\section{Trans-Golgi-like vesicles are present within the ASC filament network}

209 How NLRP3 is activated by a broad range of different stimuli - including ion fluxes, reactive 210 oxygen species and mitochondrial damage - remains an open question. A common feature of

211 NLRP3 activation by these different stimuli is that the trans-Golgi network (TGN) is dispersed, 212 and NLRP3 locally accumulates at foci within the dispersed TGN ${ }^{7,12}$. These NLRP3 foci have 213 been proposed to serve as nucleation sites for ASC and caspase-1 recruitment and activation ${ }^{7}$

214 Consistent with previous studies, we observed dispersion of the TGN in WT iBMDM and THP-1

215 cells, and in iBMDMs expressing ASC-mCerulean (Figs. 3a,b; S3a). Moreover, NLRP3 partially

216 colocalized with the TGN marker TGN38 in WT iBMDMs primed with LPS, with or without

217 nigericin stimulation (Fig. 3a). In stimulated iBMDMs, ASC-mCerulean puncta were located

218 adjacent to anti-TGN38 fluorescence, but there was little direct overlap between the two

219 markers (Fig. 3b). In our tomographic reconstructions, vesicles with diameters of 50 to $300 \mathrm{~nm}$

220 were observed within the ASC filament network (Figs. 3c,d; S2g; S3b; Movies S3, S4). We

221 observed similar vesicles within the filament network in chemically fixed ASC-mCerulean puncta

222 imaged by room temperature electron tomography (Fig. S3c-e). Notably, these vesicles had

223 low-density lumens, a feature characteristic of TGN vesicles ${ }^{38}$. Ribosomes were also found 
224 embedded throughout the ASC filament network. Ring-shaped densities were occasionally

225 observed within tomograms of ASC puncta, but the resolution of the reconstructions was

226 insufficient to infer the composition of these features (Fig. 1d). Together, our cryo-ET and

227 fluorescence microscopy data supports the model that the dispersed TGN may serve as a

228 platform for ASC recruitment to multiple NLRP3 foci, which nucleate ASC polymerization, with

229 subsequent branching of ASC filaments driving growth into a micron-scale punctum.

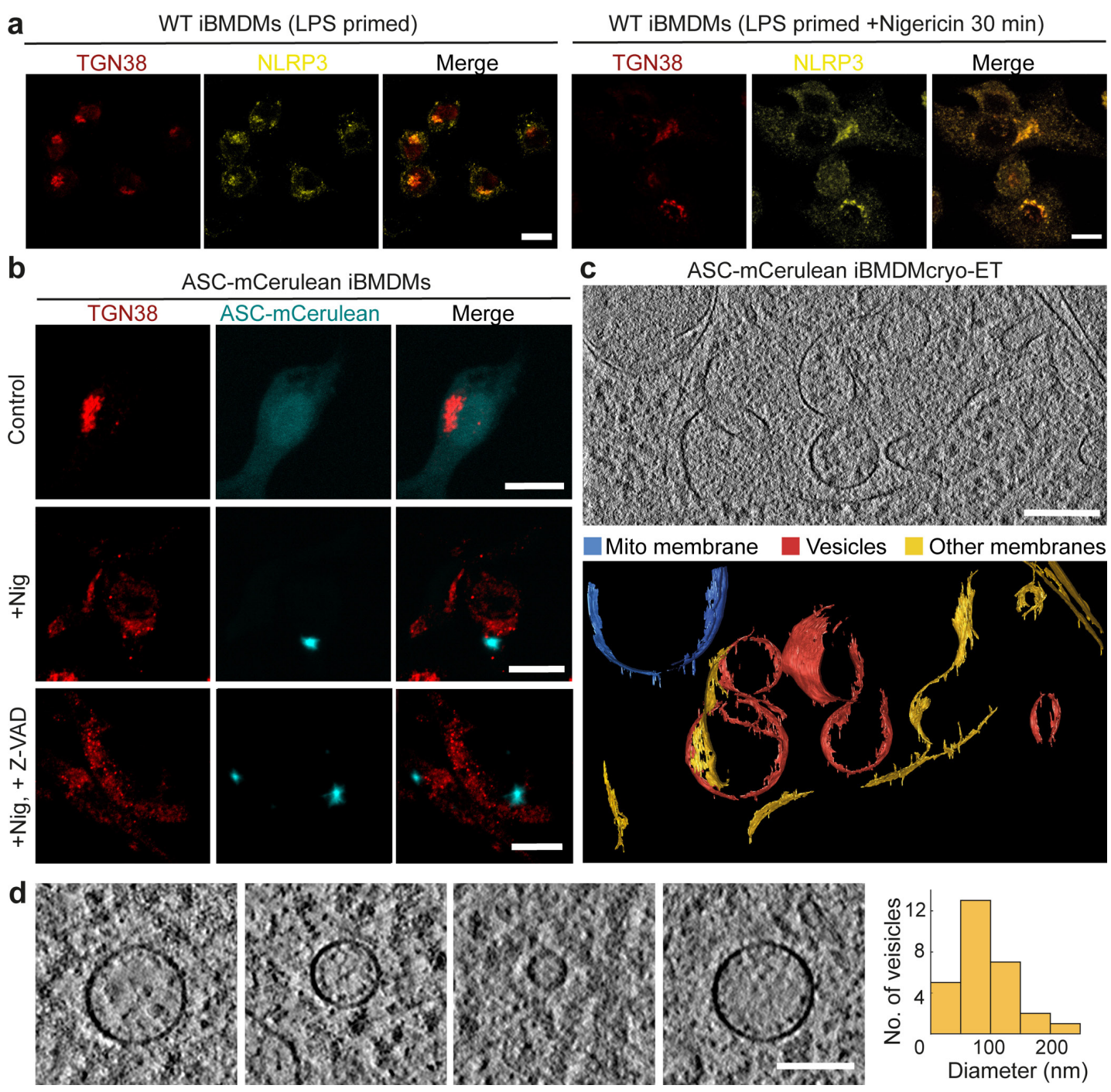

232 (a) Immunofluorescence microscopy of WT iBMDMs primed with LPS or stimulated with

233 nigericin. NLRP3 partially colocalized with TGN38. The TGN was dispersed following

234 stimulation. Scale bar, $10 \mu \mathrm{m}$. 
235 (b) Immunofluorescence microscopy of iBMDMs expressing ASC-mCerulean before and after 236 stimulation with nigericin, or nigericin and caspase inhibitor Z-VAD-FMK. ASC-mCerulean 237 puncta were adjacent to anti-TGN38 fluorescence, but there was little overlap between the 238 two markers. Scale bar, $10 \mu \mathrm{m}$.

239 (c) 1.36-nm thick virtual tomographic slices of vesicles within the ASC-mCerulean filament 240 network in an iBMDM. Scale bar, $200 \mathrm{~nm}$. Lower panel, 3-D segmentation model of the 241 same volume.

242 (d) Gallery of vesicles from tomographic reconstructions of cryo-FIB-milled ASC-mCerulean 243 iBMDMs. Scale bar, $100 \mathrm{~nm}$. The histogram shows the vesicle diameter distribution.

Since NLRP3 inflammasome activation was reported to require transport of NLRP3 to 245 the MTOC ${ }^{12,13}$, we examined the localization of ASC/caspase-1 puncta relative to the MTOC. 246 Fluorescent markers for ASC and caspase-1 did not colocalize with the MTOC components $\gamma$ -

247 tubulin, pericentrin or ninein in WT or ASC-mCerulean iBMDMs stimulated with LPS and 248 nigericin (Fig. S4a,b). In stimulated THP-1 cells, ASC/caspase-1 puncta were on average closer 249 to the MTOC, with one quarter of puncta within $2 \mu \mathrm{m}$ of the MTOC, but there was little direct 250 overlap between the fluorescent markers (Fig. S4c).

\section{Aberrant mitochondrial morphology during pyroptosis visualized by cryo-ET}

252 Mitochondria function as a nexus for multiple pathogen-sensing and damage-sensing signalling 253 pathways. Innate immune sensors of viral nucleic acids ${ }^{39}$ and mitochondrial damage converge 254 on the outer mitochondrial membrane, and induce apoptosis or pyroptosis, depending on the 255 specific danger signal that is sensed ${ }^{40,41}$. We observed fragmentation of the filamentous network 256 of mitochondria in ASC-mCerulean iBMDMs following stimulation with LPS and nigericin, similar 257 to AIM-2 dependent mitochondrial remodelling ${ }^{41}$, even prior to ASC punctum formation (Fig.

258 4a). Mitochondrial remodelling coincided with dissipation of the membrane potential (Fig. 4b,

259 Fig. S5a). To visualize and analyse the morphology of mitochondria soon after inflammasome 
260 activation we performed cryo-ET reconstructions of mitochondria following LPS priming and

261 nigericin stimulation to induce ASC speck formation. In LPS primed iBMDMs expressing ASC-

262 mCerulean, a 30-min stimulation with nigericin resulted in mitochondria with a more rounded

263 overall shape and smaller size than in unstimulated cells (Fig. 4c). Cryo-ET reconstructions and

264 segmentation models of WT iBMDMs 1-h post-stimulation showed that the mitochondria in

265 these cells had an abnormal ultrastructure, with the inner membranes forming concentric tubular

266 structures instead of the regularly spaced lamellar cristae observed in unstimulated cells (Fig.

267 4c,d). To quantify the changes in mitochondrial morphology associated with ASC-dependent

268 signalling, four parameters were extracted and measured using Fiji ${ }^{42}$ : cristae spacing, inner-to-

269 outer membrane spacing, cristae lumen width and cristae apex angles (Fig. 5a,b). We found the

270 cristae spacing to be two- to fourfold smaller in pyroptotic mitochondria than in healthy

271 mitochondria. The other three parameters were not significantly different. 

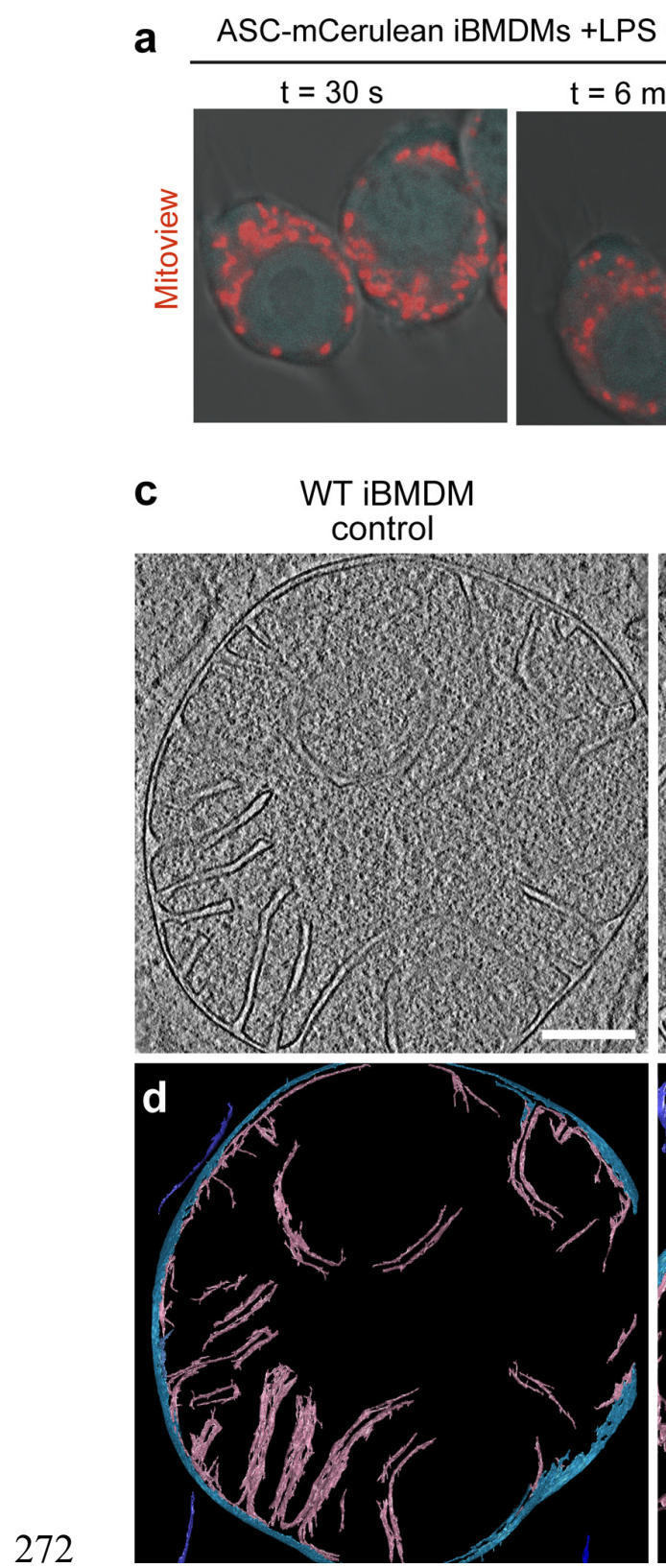

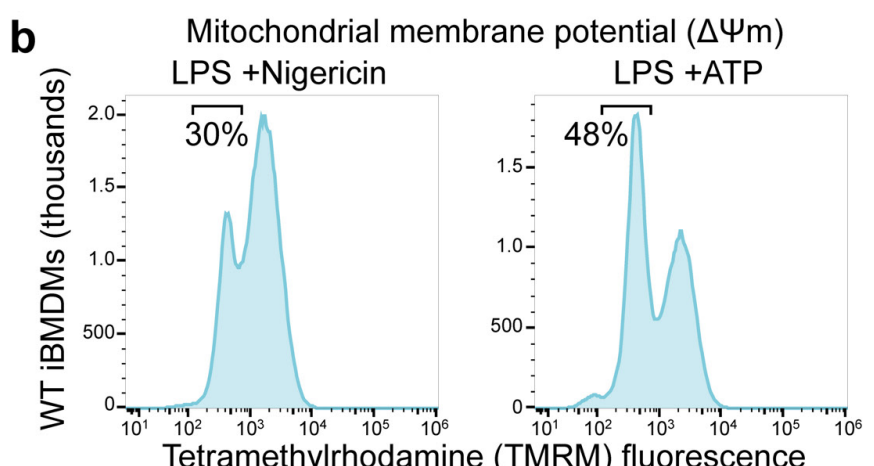

Tetramethylrhodamine (TMRM) fluorescence

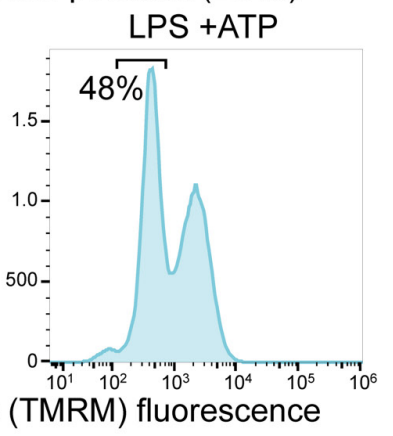

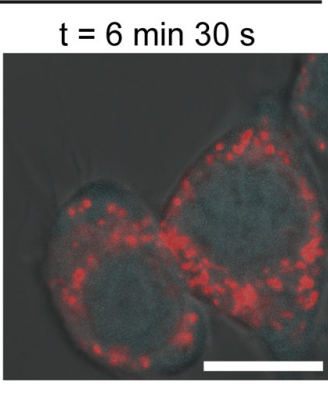

ASC iBMDM + LPS +
nigericin (30 min)
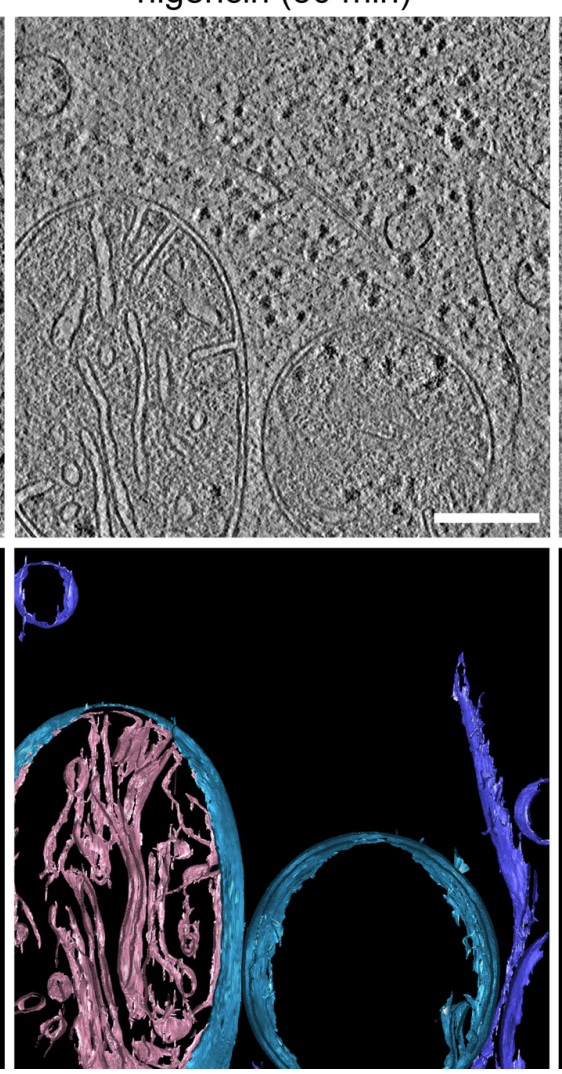

WT iBMDM +LPS+ nigericin $(1 \mathrm{~h})$
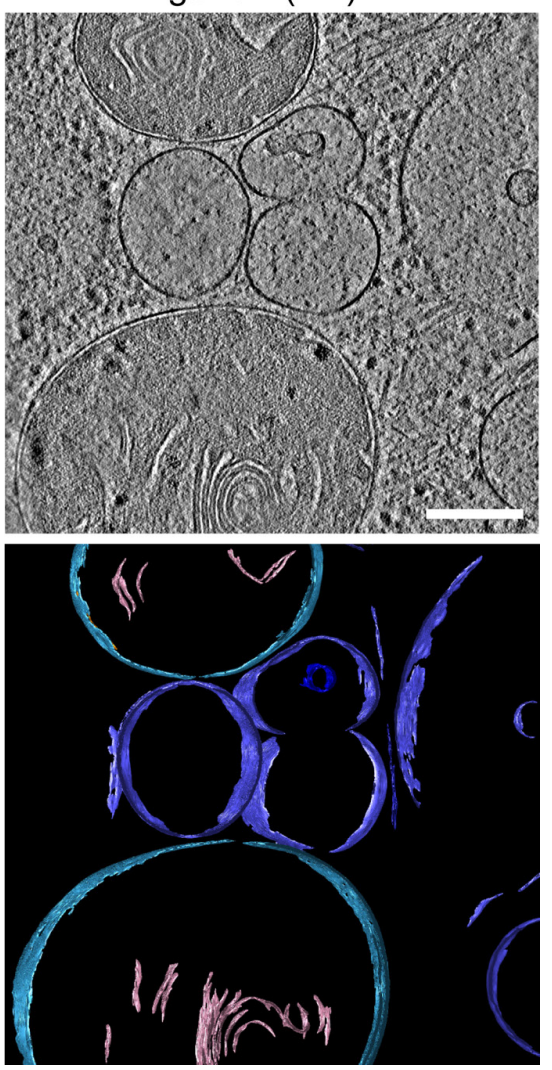

273 Figure 4. Remodelling of mitochondrial cristae after activation of NLRP3 inflammasome.

274 (a) Snapshots of live ASC-mCerulean iBMDMs labelled with a MitoView mitochondrial dye.

275 Overlaid fluorescence and bright-field images show soon fragmentation of the mitochondrial

276 network prior to ASC-speck formation. Scale bar $10 \mu \mathrm{m}$.

277 (b) Flow cytometry histogram of iBMDMs stained with tetramethylrhodamine (TMRM), a dye

278 sensitive to mitochondrial membrane potential, $\Delta \Psi \mathrm{m}$. Cells were stimulated with LPS and

279 nigericin (left), or LPS and ATP (right) for $30 \mathrm{~min}$. 
280 (c) Reconstructed cryo-ET tomographic slices of an unstimulated iBMDM (left); ASC-mCerulean

281 iBMDMs stimulated with LPS and nigericin for 30 min (centre); or WT iBMDMs, stimulated

282 with LPS and nigericin for $1 \mathrm{~h}$ (right).

283 (d) 3-D segmentation models of the outer and inner envelope membranes (purple) and cristae

284 membranes (pink) in the tomographic slices shown immediately above in (c).

\section{Discontinuities in the outer membranes of mitochondria during pyroptosis}

286 During apoptosis, the Bax and Bak proteins create large disruptions in the outer mitochondrial

287 membrane ${ }^{40,43}$. Our cryo-ET reconstructions showed that mitochondria in pyroptotic iBMDMs

288 stimulated with LPS/nigericin lacked any such large outer-membrane disruptions. We did,

289 however, identify small discontinuities in the outer mitochondrial membrane in some of the

290 stimulated ASC-mCerulean and WT iBMDMs (Fig. 5c,d). The size of these gaps varied from 10

291 to $22 \mathrm{~nm}$ (Fig. S5b). The presence of an intact inner membrane adjacent to the gap in the outer-

292 membrane implied that the gap was not due to the missing wedge effect or the anisotropic

293 resolution of cryo-ET tomograms ${ }^{44}$.

The presence of 10-22-nm gaps in the outer membranes of mitochondria during

295 pyroptosis raises the question of whether these gaps cause mitochondrial outer membrane

296 permeabilization (MOMP) during inflammasome stimulation. To address this question, we

297 measured the cryo-EM density in the intermembrane space and in an adjacent cytosolic area,

298 where outer-membrane gaps were present. Unexpectedly, we found that the density at sites

299 where outer membrane gaps were present was higher than in the cytosol, indicating an increase

300 in macromolecular density at gap sites (Fig. 5e,f). In contrast, at sites where both membranes

301 were intact the intermembrane space had a lower density than the cytosol, as expected. The

302 increased cryo-ET density at gap sites spanned $10-22 \mathrm{~nm}$ along the Z-axis, matching the

303 dimensions of the gaps in $\mathrm{X}$ and $\mathrm{Y}$. We conclude that protein-rich disruptions of the outer

304 mitochondrial membrane $10-22 \mathrm{~nm}$ in diameter form during pyroptosis. 

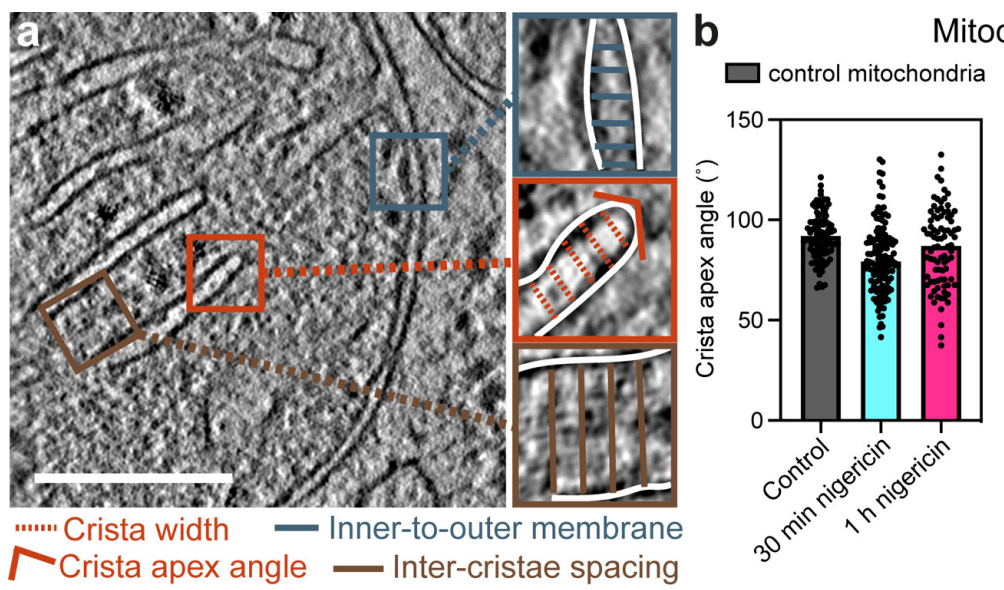

Mitochondrial morphology

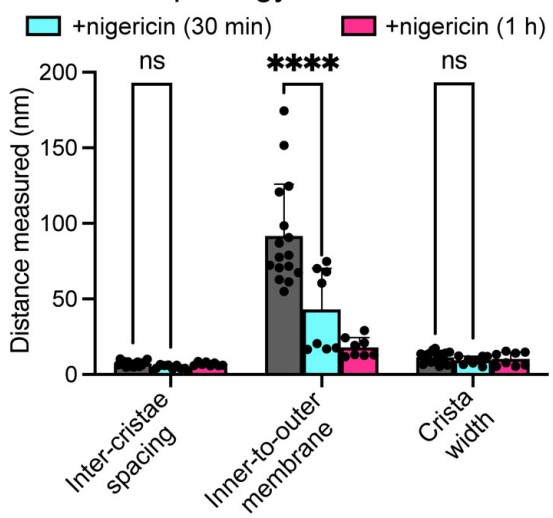

C ASC-mCerulean iBMDM
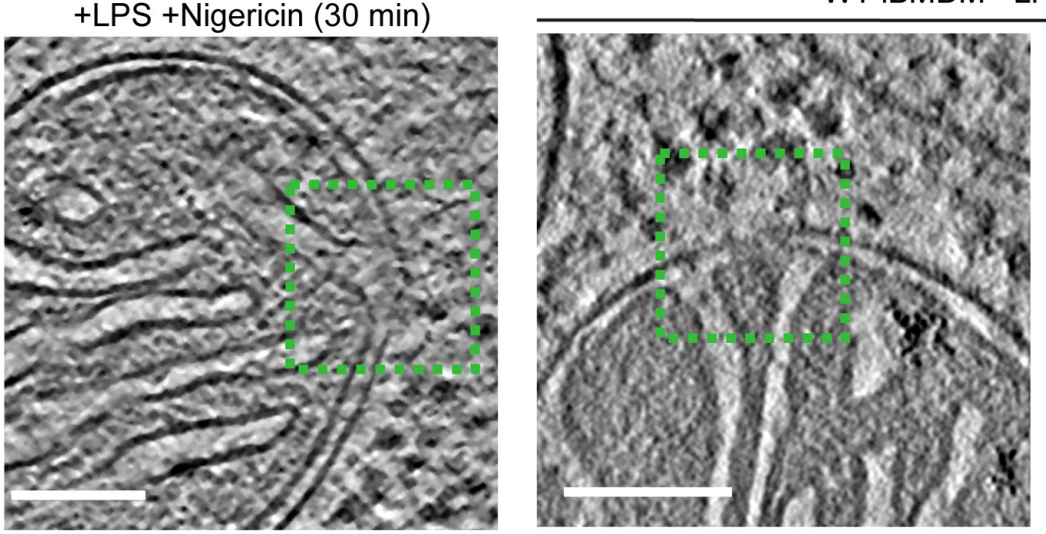

WT iBMDM +LPS +Nigericin (1 h)
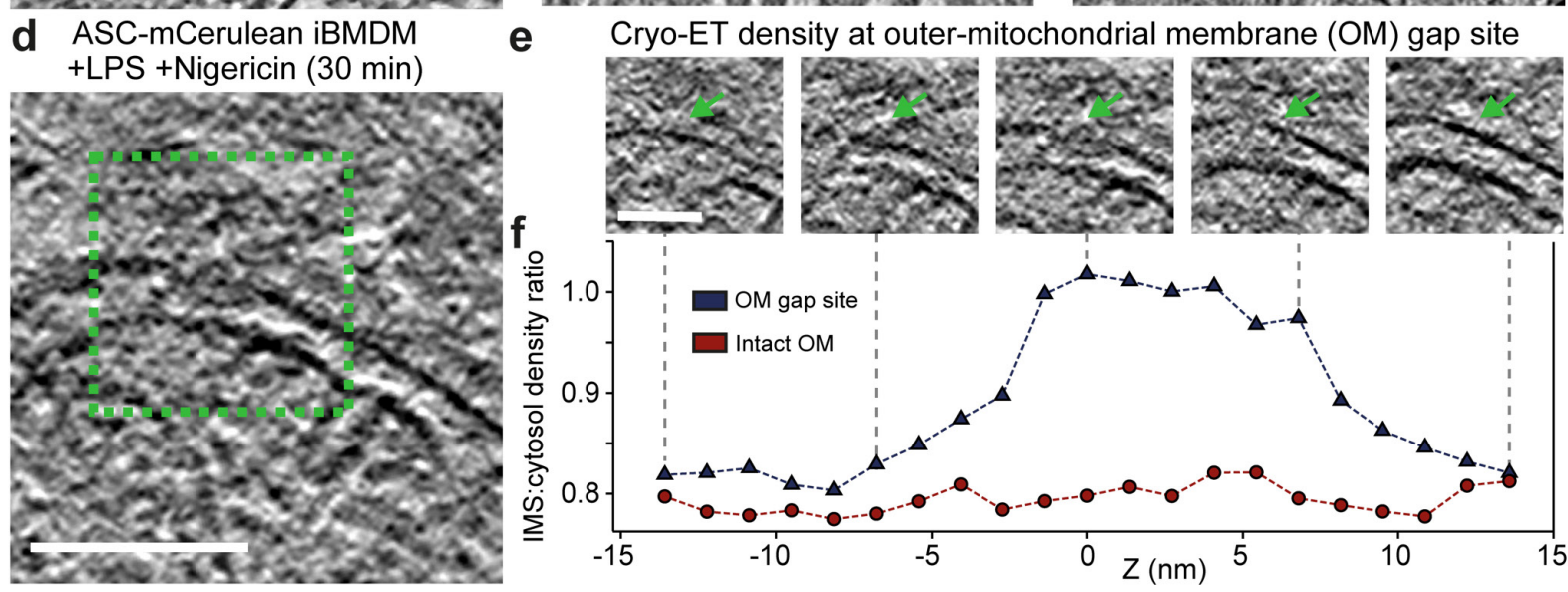

Figure 5. Outer mitochondrial membrane discontinuities in pyroptotic cells.

307 (a) Cryo-ET reconstruction of a mitochondrion in an unstimulated WT iBMDM with closeup

308 panels defining the following morphological parameters: inner-to-outer membrane spacing,

309 inter-cristae spacing, crista width and crista apex angle.

310 (b) Quantitative analysis of the morphological parameters quantified in (a) in 3-D segmented

311 surfaces of the mitochondrial membranes, as shown in (Fig. 4d). Error bars represent 
312 standard deviation between measurements. Parameters were measured from four

313 tomograms for the control and two tomograms for each of the nigericin-stimulated samples.

314 Error bars represent standard deviation from the mean. For the crista apex angles: "control",

$315 \mathrm{n}=327 ;$ “30 min”, $\mathrm{n}=187 ;$ " $1 \mathrm{~h}$ ”, $\mathrm{n}=96$. For other parameters: "control”, $\mathrm{n}=16$; “30 min”, $\mathrm{n}$

$316=8 ;$ " $1 \mathrm{~h}$ ", $\mathrm{n}=8$. Statistical test: two-way ANOVA, ns, $\mathrm{P}>0.05 ;{ }^{* \star \star *}, \mathrm{P}<10^{-4}$. See Dataset

317 S3 for source data.

318 (c) Cryo-ET reconstructions of mitochondria in ASC-mCerulean iBMDMs stimulated with

319 nigericin for 30 min (left) and in WT iBMDMs stimulated with nigericin for $1 \mathrm{~h}$ (centre, right).

320 Dashed boxes denote outer-membrane discontinuities. Scale bars, $100 \mathrm{~nm}$.

321 (d) Higher magnification view of a mitochondrial outer-membrane discontinuity (dashed box).

322 Scale bar, $100 \mathrm{~nm}$.

323 (e) Z-stack series for the boxed region in (d). Arrows mark the outer-membrane discontinuity.

$324 \quad$ Scale bar, $20 \mathrm{~nm}$.

325 (f) Quantitative analysis of the cryo-ET density at the outer-membrane (OM) gap site in the Z-

326 stack series shown in (e), and at a site in the same tomogram with intact inner and outer

327 membranes. The graph plots the average pixel density ratio $<\rho($ IMS $)>\mid<\rho($ cyt $)>$ for each Z-

328 slice, where $<\rho($ IMS $)>$ and $<\rho($ cyt $)>$ are the average pixel densities of circular areas $35.4 \mathrm{~nm}$

329 in diameter, in the mitochondrial intermembrane space (IMS) and an adjacent cytosolic

$330 \quad$ area, respectively. See Dataset S2 for source data.

\section{Discussion}

332 NLRP3 inflammasome signalling assemblies have long been known from fluorescence

333 microscopy imaging to form puncta, or specks, containing ASC and caspase-1. Room

334 temperature electron tomography of ASC puncta in fixed zebrafish sections ${ }^{26}$ and iBMDMs (Fig.

335 S3d) showed a dense filament network within the punctum, but the chemical fixation and

336 staining agents precluded further ultrastructure from being resolved. Our cryo-ET 
337 reconstructions, obtained in unstained and fully hydrated conditions, show that the speck is

338 formed of a filamentous network consisting of hollow-tube branched filaments with the

339 dimensions predicted for ASC filaments, based on structural studies of purified ASC PYD

340 filaments and full-length monomeric $\mathrm{ASC}^{23,24}$. The identification of a filamentous network

341 containing mCerulean-labelled ASC positively identifies the protein as the principal filament-

342 forming component in the puncta. These data are supported by analysis of similar structures in

343 wild-type cells labelled with a fluorescent caspase 1 substrate. The filament structure of the

344 ASC with an mCerulean tag on the C-terminus supports the model whereby the PYD forms the

345 filament core, and the CARD decorates the filament. The extensive filament branching visible in

346 the reconstructions is thought to depend on CARD-CARD interactions because the CARD is

347 required for puncta formation, whereas the ASC PYD alone forms unbranched filaments that

348 lack the structural stability that is inherent to a branched network ${ }^{17,18,23-26}$.

The density of ASC filaments varied in the cryo-ET reconstructions, with multiple core

350 regions of densely packed filaments separated by sparser regions. This suggests that the

351 higher-order structure of the punctum was likely seeded by multiple oligomeric assemblies,

352 rather than growing concentrically from a single nucleation site. In contrast with huntingtin and

353 other neurotoxic protein aggregates, which exclude other macromolecules based on cryo-ET

354 reconstructions ${ }^{45}$, the structural organisation of the ASC filament network allows ribosomes and

355 small TGN-like vesicles to be retained in the puncta, or to permeate through them. Permeability

356 of the ASC network to macromolecules could be important for efficient recruitment and release

357 of effector, substrate and product molecules. Permeability to vesicles would allow active

358 transport of TGN vesicles carrying activated inflammasome seed-oligomers from sites of NLRP3

359 activation to the site of punctum formation, as recently proposed ${ }^{12}$. Moreover, the ribosomes

360 within the puncta could potentially contribute to the pyroptotic signalling programme, for

361 example by expressing proteins required for signalling, or by responding to danger signals. 
362 Indeed, NLRP3 signalling can be activated by translational arrest through direct and indirect 363 mechanisms ${ }^{46}$, including binding of fungal polysaccharides to ribosomes ${ }^{47}$. within the cell, also allowed morphometric analysis of mitochondria in the inflammasome activated cellular environment. The clearest differences between mitochondria in inflammasome

367 activated cells and unstimulated cells were a two to fourfold reduction in cristae spacing, and 368 the appearance of protein-rich discontinuities of $10-20 \mathrm{~nm}$ in diameter in the outer membrane, 369 which are distinct from BAK/BAX macropores. Permeabilization of both the plasma membrane 370 and outer mitochondrial membrane was reported previously in cells undergoing caspase-1371 dependent pyroptosis after inflammasome activation ${ }^{41}$. Pore formation in the plasma membrane 372 by the $\mathrm{N}$-terminal domain of gasdermin $\mathrm{D}$ (GSDMD) after cleavage by caspase-1 is required for 373 inflammasome-dependent pyroptosis ${ }^{48}$. The outer-membrane discontinuities in the mitochondria

374 of inflammasome-activated cells had similar dimensions to GSDMD pores, which have an 375 average inner diameter of $22 \mathrm{~nm}^{48}$ (or $13-34 \mathrm{~nm}$ based on atomic force microscopy ${ }^{49}$ ). Future 376 studies are warranted to test the hypothesis that the outer-membrane gaps observed by cryo377 ET may correspond to mitochondrial GSDMD pores.

379 integrity while allowing downstream signalling molecules to diffuse freely and bind at high 380 density within the network. Although many structural details of the NLR inflammasome signalling 381 machinery remain unclear, this study demonstrates the potential for combined cryo-CLEM and 382 cryo-ET approaches to extract detailed, hypothesis-generating ultrastructural information for 383 fully assembled innate immune signalling complexes in their cellular context. 
Mammalian cell culture. Wild-type immortalized primary bone-marrow derived macrophages (WT iBMDMs) and ASC-mCerulean iBMDMs, kindly provided by Eicke Latz (Univ. Bonn), were 387 cultured in Dulbecco's modified Eagle Medium high glucose (DMEM; Gibco, Waltham, MA, 388 USA), supplemented with $10 \%$ v/v foetal bovine serum (FBS; Gibco). Human monocytic THP-1 389 cells (European Collection of Authenticated Cell Cultures) were grown in Roswell Park Memorial 390 Institute (RPMI) 1640 Medium (Gibco), supplemented with heat inactivated 10\% v/v FBS, 10

$391 \mathrm{mM}$ HEPES pH 7.4, 1 mM Sodium Pyruvate and 0.05 beta-mercaptoethanol. Cells were 392 checked regularly for mycoplasma contamination by MycoAlert ${ }^{\mathrm{TM}}$ mycoplasma detection kit 393 (Lonza) and cells were free of mycoplasma contamination.

394 Inflammasome stimulation. For stimulation of WT iBMDMs and ASC-mCerulean iBMDMs, 395 cells were primed with $200 \mathrm{ng} / \mathrm{ml}$ LPS for $3 \mathrm{~h}$ in Opti-MEM® (Gibco) or DMEM supplemented 396 with 10\% FBS before NLRP3 stimulus was used. The following stimulus were used: $10 \mu \mathrm{M}$ 397 nigericin (Sigma or Invivogen) and $5 \mathrm{mM} \mathrm{ATP} \mathrm{(pH} \mathrm{adjusted} \mathrm{to} \mathrm{7.4,} \mathrm{Sigma)} \mathrm{for} 30$ min or $60 \mathrm{~min}$ 398 with pan-caspase inhibitor Z-VAD-FMK (Invivogen) or carboxyfluorescein-labelled inhibitor of 399 caspase-1 YVAD (FAM-FLICA, ImmunoChemistry Technologies) for in situ cryo-ET sample 400 preparation unless otherwise specified. For stimulation of THP-1 cells, priming and stimulation 401 were performed in RPMI Medium and conditions were listed above. To stain active caspase-1, 402 the supernatant was removed following inflammasome stimulation; replaced with DMEM 403 supplemented with heat-inactivated $10 \%$ v/v FBS containing $0.5 x$ reconstituted FAM-FLICA 404 (ImmunoChemistry Technologies).

405 Cytokine measurement and immunoblotting. IL-1 $\beta$ secretion was measured by ELISA as 406 previously described ${ }^{27}$. Briefly, supernatants were collected after inflammasome activation and 407 BD BioSciences OptEIA was used to measure mouse IL-1 $\beta$ concentration in cell supernatant 408 according to the manufacturer's instructions. 
$410 \mathrm{X}-100,1 \mathrm{mM}$ PMSF, $10 \mu \mathrm{g} / \mathrm{mL}$ leupeptin, $1 \mu \mathrm{g} / \mathrm{mL}$ aprotinin) on ice for $10 \mathrm{~min}$ before

411 centrifugation. Supernatant was collected and boiled in SDS sample loading buffer for 5 min.

412 Following separation by $4-20 \%$ gradient SDS-PAGE, the proteins were transferred to a PVDF

413 membrane. Membrane was blocked in 5\% milk in PBS + 0.2\% Tween-20. The primary antibody

414 was rabbit monoclonal anti-mouse GSDMD (Abcam, ab209845, RRID:AB_2783550). The

415 secondary antibody was goat anti rabbit IgG-HRP (Santa Cruz Biotechnology, sc-2004,

416 RRID:AB_631746).

417 Immunofluorescence staining. For immunofluorescence, cells were plated as monolayer on

$418 \mu$-Slide 8-Well chamber (ibiTreat, Ibidi, 80826) or 12-well chamber (removable, Ibidi, 81201).

419 Cells were primed and stimulated as above, and then fixed with $4 \%$ paraformaldehyde for 5 to

$42010 \mathrm{~min}$ at room temperature. Cells were permeabilised and blocked in $0.1 \%$ Saponin (Sigma,

42147036 ) supplemented with 20\% FBS (Gibco) or 2\% Bovine Serum Albumin (Sigma, A7030) in

422 PBS with primary antibody overnight. Washing was performed before cells were incubated with

423 secondary antibodies. The following primary antibodies were used: rabbit anti-ASC rabbit

424 polyclonal pAb AL177, 1:200 dilution (AdipoGen, AG-25B-0006, RRID:AB_2490440); rabbit

425 anti-ASC monoclonal ASC/TMS1(D2W8U), 1:800 dilution (Cell Signalling Technology, 67824,

426 RRID:AB_2799736); goat anti-NLRP3 polyclonal, 1:200 dilution (Abcam, ab4207,

427 RRID:AB_955792); goat anti-IL-1 $\beta$ polyclonal, 1:500 dilution (R\&D Systems, AF-401-NA,

428 RRID:AB_416684); mouse anti-y-Tubulin monoclonal, 1:400 dilution (Sigma-Aldrich, T6557,

429 RRID:AB_477584); rabbit anti-TGN38 polyclonal, 1:250 dilution (Novus Biologicals, NBP1-

430 03495, RRID:AB_1522533); rabbit anti-TOM20 FL-145 polyclonal, 1:80 dilution (Santa Cruz

431 Biotechnology, sc-11415, RRID:AB_2207533). The Alexa Fluor secondary antibodies (488, 555,

432568 and 647) used were: 488-labelled donkey anti-goat IgG $(H+L)(1: 500$, ThermoFisher,

433 A11055, RRID:AB_2534102); 555-labelled goat anti-rabbit $\lg G(H+L)(1: 500$, ThermoFisher, 
434 A21428 RRID:AB_141784); 568-labelled goat anti-mouse IgG $(\mathrm{H}+\mathrm{L})(1: 500$, ThermoFisher,

435 A11004, RRID:AB_2534072); 647-labelled goat anti-rabbit lgG (H+L) (1:500, ThermoFisher,

436 A21244, RRID:AB_2535812). After incubation with secondary antibodies at room temperature

437 for $1 \mathrm{~h}$, cells were washed three times with blocking buffer, one time with PBS and final wash

438 with water before mounting with ProLong Gold Antifade Mountant with DAPI (ThermoFisher).

439 Confocal fluorescence imaging. Single point-scanning confocal microscopy was carried out

440 on a Zeiss (Oberkochen, Germany) LSM 780 or LSM 710 microscope using a 40x/1.3 NA Fluar

441 or $63 x / 1.4$ NA Plan-apochromat oil immersion objective lens. The microscope was equipped

442 with 405, 458, 488, 514, 561 and $633 \mathrm{~nm}$ laser lines. Multi point-scanning confocal microscopy

443 was carried out on Visitech (Sunderland, UK) iSIM mounted on a Nikon (Tokyo, Japan) Ti2

444 microscope stand using a 100x/1.49 NA SR Apo TIRF oil immersion objective lens or a 60x/1.2

445 NA Plan Apo VC water immersion objective lens. The iSIM was equipped with 405, 445, 488,

446561 and $640 \mathrm{~nm}$ laser lines and Hamamatsu (Hamamatsu, Japan) ORCA-Flash4.0 V3 sCMOS

447 cameras. Filter ranges: green (500-545 nm), red (593-624 nm). Live-cell samples were heated

448 to $37^{\circ} \mathrm{C}$ and supplemented with $5 \% \mathrm{CO}_{2}$ using a microscope incubation chamber.

449 Live-cell imaging and Fluorescence Recovery after Photobleaching (FRAP). ASC-

450 mCerulean iBMDMs were primed and induced as previously described with the presence of

451 caspase-1 inhibitor (Z-VAD-FMK). Confocal FRAP was performed on a Zeiss LSM 710

452 microscope equipped with a 63x/1.4 NA Plan-apochromat oil immersion objective lens and a

$453458 \mathrm{~nm}$ laser line for excitation and bleaching. The sample environment was heated to $37^{\circ} \mathrm{C}$ and

454 supplemented with $5 \% \mathrm{CO}_{2}$ using a microscope incubation chamber. ASC-mCerulean speck

455 was photobleached with $100 \%$ laser power. Images were acquired at $3 \mathrm{~s}$ intervals. Images were

456 collected at three pre-bleach timepoints and for $160 \mathrm{~s}$ post-bleaching. Movies were analysed in

457 ImageJ/Fiji ${ }^{42}$ using a customised script (https://github.com/jboulanger/imagej-

458 macro/tree/main/FRAP Measure). Briefly, measures were normalised to account for the general 
459 photobleaching caused by image acquisition as well as sample motion over time. Normalised

460 fluorescence intensity measurements were obtained after background and bleaching

461 corrections. The timeframe varied for experiments $(n=8)$. Each measurement was interpolated

462 using the normalised fluorescence intensity measurements and imported to Python for plotting

463 of the fluorescence intensity curve.

464 Quantification of Mitochondrial membrane potential. After 30 min of inflammasome

465 stimulation as described above, iBMDMs were incubated with 20 nM TMRM (Life Technologies)

466 for $15 \mathrm{~min}$. iBMDMs were incubated for $3 \mathrm{~h}$ in $50 \mu \mathrm{M}$ of the mitochondrial uncoupler carbonyl

467 cyanide 3-chlorophenylhydrazone (CCCP; ThermoFisher, M20036). Control samples included:

468 untreated; TMRM+; TMRM+CCCP+; TMRM+LPS+ iBMDMs. TMRM fluorescence was

469 quantified by flow cytometry and data were acquired on Eclipse flow cytometer (Sony

470 Biotechnology). Quantification was set at 100,000 for cell count and a spectrum window of (FL3

471 (595BP)) was used to detect TMRM signal. Data were analysed and visualised using Flowjo10

472 (TreeStar, Ashland, Oregon, USA).

473 Cryo-ET sample preparation. Quantifoil finder grids (R2/1 or R2/2, Au 200 mesh grid,

474 Quantifoil Micro Tools, Germany) were glow discharged with a $30 \mathrm{~mA}$ current for $30 \mathrm{~s}$ using

475 Edwards Sputter Coater (S150B). Then grids were sterilized by UV irradiation for 10 min and

476 immersed in PBS supplemented with fibronectin solution (10 $\mu \mathrm{g} / \mathrm{ml}$, Sigma) overnight in 8-well

477 or coculture wells (ibidi). Grids were washed with PBS three times. Cells were seeded in 8-well

478 or coculture wells (ibidi) and incubated overnight at $37^{\circ} \mathrm{C}$ and $5 \% \mathrm{CO}_{2}$. Cells cultivated on grids

479 were primed, induced with inflammasome stimuli and were plunge-frozen in liquid ethane using

480 Leica EM GP2 cryo-plunger. Prior to plunging, $4 \mu \mathrm{l}$ of cell culture medium was added to the cell

481 side and backside blotting was applied for 6-8 s. The chamber conditions were maintained at

$48237^{\circ} \mathrm{C}, 100 \%$ humidity during freezing. Grids were stored in liquid nitrogen. 
Cryo-fluorescent light microscopy (Cryo-FM) and cryo-focused ion-beam (FIB) milling.

484 Grids were screened for cells with ASC/caspase-1 speck by light microscopy using a cryo-FM

485 microscope (Leica EM Cryo CLEM, Leica Microsystems), equipped with a cryo-stage, an

486 ORCA-Flash4.0 V2 sCMOS camera (Hamamatsu Photonics) and a 50x/0.9 NA HCX PL APO

487 cryo-objective lens. Montage acquisition of grids was performed with Leica LAS X software,

488 while recording the following channels: green (L5 filter, $50 \mathrm{~ms}$ ), far red (Y5 filter, $20 \mathrm{~ms}$ ), and

489 brightfield $(50 \mathrm{~ms})$. Z-stacks were recorded at $0.5 \mu \mathrm{m}$ with step size 21 for each grid square to

490 determine the best focus for the montage. The montage was completed by stitching the best

491 focus image from the Z-stack with Leica LAS X.

Lamellae were prepared using a Scios Dual Beam FIB scanning electron microscope

493 (SEM; ThermoFisher) equipped with a Quorum cryo-stage (PP3010T). The milling protocol was

494 adapted from previously published method ${ }^{29}$. Grids were coated with organometallic platinum

495 using the gas injection system (GIS; ThermoFisher) operated at RT, for $8 \mathrm{~s}$, at $12 \mathrm{~mm}$ working

496 distance and $25^{\circ}$ stage tilt. A first rough milling was performed at $25^{\circ}$ stage tilt with a $30 \mathrm{kV}$ ion

497 beam voltage and $1 \mathrm{nA}$ current until the lamella thickness reached $10 \mu \mathrm{m}$. The micro-expansion

498 joints were applied to improve lamella stability ${ }^{32}$ using the milling parameters listed above. Then

499 stage was tilted to $20^{\circ}$ for all subsequent milling steps. Rough milling steps were applied as

500 following with $30 \mathrm{kV}$ ion beam voltage: a $5 \mu \mathrm{m}$ lamella thickness was reached with current 0.5

$501 \mathrm{nA} ; 3 \mu \mathrm{m}$ lamella thickness was reached with a $0.3 \mathrm{nA}$ current; and $1 \mu \mathrm{m}$ lamella thickness was

502 obtained with a $0.1 \mathrm{nA}$ current. Fine milling to a final lamella thickness of approximately $200 \mathrm{~nm}$

503 was performed with ion beam settings of $30 \mathrm{kV}$ and $50 \mathrm{pA}$ or $10 \mathrm{pA}$, or $10 \mathrm{kV}$ and $23 \mathrm{pA}$ or 11

$504 \mathrm{pA}$.

505 Grids were loaded to a Linkam cryo-stage (CMS196V), and lamellae were imaged on a

506 Zeiss microscope equipped with Axiocam 503 mono and Colibri 7-illumination module R(G/Y)

507 CBV-UV. Z-stacks of the lamellae were acquired to identify ASC/caspase-1 specks using a 200- 
$300 \mathrm{~nm}$ step size. A projected cryo-fluorescent image was used to correlate with an SEM image using the EC-CLEM plugin from $\mathrm{ICY}^{36}$. Registration between the lamella map and SEM image was achieved by transforming coordinates using edges or salient features of the lamella. The

511 maximum projection Z-stack was then saved for correlation with SerialEM ${ }^{32,33}$.

512 Tilt-series acquisition and tomogram reconstruction. Grids with a fluorescent lamella were

513 transferred to a $300 \mathrm{kV}$ Titan Krios electron microscope (ThermoFisher) equipped with an

514 energy filter (Gatan). Movies were acquired with a K2 or K3 direct electron detector with

515 SerialEM ${ }^{33,34}$. Cryo-FM/EM correlation was then used to locate an area of interest for tilt-series

516 acquisition. Briefly, a medium-magnification montage EM map (MMM) of the lamella was

517 generated and a fluorescence map (FM) was loaded into SerialEM. The maps were correlated

518 through recognition of geometric edges of the lamella. Transformation of coordinates yielded an

519 overlay map which provided guidance for tilt-series acquisition. Tilt-series were collected at a

520 nominal $32,000 x$ or $29,000 x$ magnification, resulting in pixel size $3.4 \AA$ or $2.7 \AA$, over a tilt range

521 of $-60^{\circ}$ to $+60^{\circ}$ with $1^{\circ}$, or $2^{\circ}$ increments, a total dose of $140-240$ electrons $/ A^{2}$ and a nominal

522 defocus range of -4 to $-8 \mu \mathrm{m}$. A dose-symmetric scheme was used ${ }^{50}$. A pre-processing script

523 was used from SubTOM (by Dustin Morado; https://github.com/DustinMorado/subTOM).

524 Frames were aligned using IMOD ${ }^{37}$. Reconstruction was performed by weighted back-

525 projection, and segmentation with IMOD. The MATLAB script deconv from Warp ${ }^{51}$ was used for

526 visualisation. Filament tracing was performed manually with IMOD and UCSF Chimera ${ }^{52}$.

527 Membrane segmentation was performed with TomoSegMemTV ${ }^{53}$.

528 Quantifications and statistical analysis. Filament lengths were measured using IMOD $^{37}$ from 529 six tomograms of ASC-mCerulean iBMDMs and one tomogram of WT iBMDMs labelled with 530 FAM-FLICA. Histograms were plotted with MATLAB. Branching angles were measured with $531 \mathrm{Fiji}^{42}$ and exported to MATLAB for plotting. The filament diameter was determined in Fiji by 532 measuring density profiles of 20-nm lines perpendicular to the ASC filament (15 pixels in width, 
533 ASC-mCerulean tomogram with13.6 A/pixel and WT ASC tomogram with $10.78 \AA$ Apixel). The

534 grey value profiles were normalized to their maximal individual value. Normalized grey profile

535 were aligned and exported to Python for plotting. Similarly, diameters of trans-Golgi-like vesicles

536 were measured in Fiji and exported to MATLAB for plotting.

Mitochondrial tomograms were loaded in $\mathrm{Fiji}^{42}$ and ROI manager was used to measure

538 morphological parameters, which were compared using Two-way ANOVA and plotted with

539 GraphPad Prism. For the inner-to-outer membrane spacing, the distance between the nearest

540 edges of the inner and outer membranes was measured. The cristae width was measured

541 between the inner membranes of the cristae. The spacing between cristae were measured

542 using the perpendicular distance between the internal border of the neighbouring cristae. The

543 inner-to-outer membrane spacing, cristae width and inter-cristae spacing were measured

544 perpendicular to the membranes, evenly spaced for each tomogram, with thirty values per slice,

545 for four slices per tomogram, from eight tomograms. The cristae apex angles were measured for

546 every tip visible in ten slices per tomogram. Every value was plotted along with the average. If

547 the end of a crista was flat, then the measurement of the two angles on either side was

548 measured. Any measurement was only taken if the internal border of the membrane was clearly

549 distinguishable from the matrix. All measurements were evenly spaced through each slice, to

550 give a value representative of the whole slice, using all the mitochondria present in each image.

551 All measurements were taken using 8 different tomograms: four control, two 30-min nigericin-

552 treated, and two 60-min nigericin-treated mitochondria.

For measurement of the outer-mitochondrial membrane gap density, we calculated the

554 density ratio as follows. A total of 21 virtual slices were selected in a region containing a visible

555 outer-membrane gap. The average pixel grey value was calculated from circular areas $13.6 \mathrm{~nm}$

556 in diameter: three cytosolic areas, three inter-membrane space areas and an outer-membrane

557 gap area. This was repeated for each of the 21 virtual slices. The ratio of average outer- 
558 membrane gap site to cytosolic grey value, and the ratio of average inter-membrane space site

559 to cytosolic grey value were calculated for each virtual slice. The grey area ratio was then

560 plotted with Igor (WaveMetrics, Inc.) to show the change of protein density in Z direction.

\section{Data availability}

562 Representative electron tomograms were deposited in EMDB with codes EMDB with codes

563 EMD-13585, EMD-13586, EMD-135XX and EMD-135XX. Other data are available from the

564 corresponding authors upon request.

\section{Code availability}

566 SubTOM scripts and instructions are available from Github

567 [https://github.com/DustinMorado/subTOM]. The FRAP ImageJ macro is available on Github 568 [https://github.com/jboulanger/imagej-macro/tree/main/FRAP_Measure].

\section{$570 \quad$ References}

5711 Xue, Y., Enosi Tuipulotu, D., Tan, W. H., Kay, C. \& Man, S. M. Emerging Activators and

572 Regulators of Inflammasomes and Pyroptosis. Trends Immunol 40, 1035-1052,

573 doi:10.1016/j.it.2019.09.005 (2019).

5742 Karczewski, K. J. et al. The mutational constraint spectrum quantified from variation in

$575 \quad$ 141,456 humans. Nature 581, 434-443, doi:10.1038/s41586-020-2308-7 (2020).

5763 Landrum, M. J. et al. ClinVar: improvements to accessing data. Nucleic Acids Res 48,

577 D835-D844, doi:10.1093/nar/gkz972 (2020).

5784 Gong, T., Liu, L., Jiang, W. \& Zhou, R. DAMP-sensing receptors in sterile inflammation and 579 inflammatory diseases. Nature Reviews Immunology 20, 95-112, doi:10.1038/s41577-019$580 \quad 0215-7(2020)$ 
5815 Heneka, M. T., McManus, R. M. \& Latz, E. Inflammasome signalling in brain function and

582 neurodegenerative disease. Nature Reviews Neuroscience 19, 610-621,

583 doi:10.1038/s41583-018-0055-7 (2018).

5846 Swanson, K. V., Deng, M. \& Ting, J. P. The NLRP3 inflammasome: molecular activation

585 and regulation to therapeutics. Nat Rev Immunol 19, 477-489, doi:10.1038/s41577-019-

$586 \quad 0165-0(2019)$.

5877 Chen, J. \& Chen, Z. J. PtdIns4P on dispersed trans-Golgi network mediates NLRP3

588 inflammasome activation. Nature 564, 71-76, doi:10.1038/s41586-018-0761-3 (2018).

5898 Shen, C., Sharif, H., Xia, S. \& Wu, H. Structural and mechanistic elucidation of

590 inflammasome signaling by cryo-EM. Current Opinion in Structural Biology 58, 18-25,

591 doi:10.1016/j.sbi.2019.03.033 (2019).

5929 Diebolder, C. A., Halff, E. F., Koster, A. J., Huizinga, E. G. \& Koning, R. I. Cryoelectron

593 Tomography of the NAIP5/NLRC4 Inflammasome: Implications for NLR Activation.

594 Structure 23, 2349-2357, doi:10.1016/j.str.2015.10.001 (2015).

59510 Zhang, L. et al. Cryo-EM structure of the activated NAIP2-NLRC4 inflammasome reveals

596 nucleated polymerization. Science 350, 404-409, doi:10.1126/science.aac5789 (2015).

$59711 \mathrm{Hu}$, Z. et al. Structural and biochemical basis for induced self-propagation of NLRC4.

598 Science 350, 399-404, doi:10.1126/science.aac5489 (2015).

59912 Magupalli, V. G. et al. HDAC6 mediates an aggresome-like mechanism for NLRP3 and

600 pyrin inflammasome activation. Science 369, doi:10.1126/science.aas8995 (2020).

$60113 \mathrm{Li}, \mathrm{X}$. et al. MARK4 regulates NLRP3 positioning and inflammasome activation through a 602 microtubule-dependent mechanism. Nat Commun 8, 15986, doi:10.1038/ncomms15986 $603 \quad$ (2017).

60414 Oroz, J., Barrera-Vilarmau, S., Alfonso, C., Rivas, G. \& de Alba, E. ASC Pyrin Domain Self605 associates and Binds NLRP3 Protein Using Equivalent Binding Interfaces. Journal of 606 Biological Chemistry 291, 19487-19501, doi:10.1074/jbc.M116.741082 (2016). 
60715 Stehlik, C. et al. Apoptosis-associated speck-like protein containing a caspase recruitment

608 domain is a regulator of procaspase-1 activation. J Immunol 171, 6154-6163,

609 doi:10.4049/jimmunol.171.11.6154 (2003).

61016 Proell, M., Gerlic, M., Mace, P. D., Reed, J. C. \& Riedl, S. J. The CARD plays a critical role 611 in ASC foci formation and inflammasome signalling. The Biochemical journal 449, 613-621, 612 doi:10.1042/BJ20121198 (2013).

61317 Dick, M. S., Sborgi, L., Rühl, S., Hiller, S. \& Broz, P. ASC filament formation serves as a 614 signal amplification mechanism for inflammasomes. Nature Communications 7, 11929, 615 doi:10.1038/ncomms11929 (2016).

61618 Sahillioglu, A. C., Sumbul, F., Ozoren, N. \& Haliloglu, T. Structural and dynamics aspects of 617 ASC speck assembly. Structure 22, 1722-1734, doi:10.1016/j.str.2014.09.011 (2014).

61819 Matyszewski, M. et al. Cryo-EM structure of the NLRC4(CARD) filament provides insights 619 into how symmetric and asymmetric supramolecular structures drive inflammasome assembly. J Biol Chem 293, 20240-20248, doi:10.1074/jbc.RA118.006050 (2018).

62120 Gong, Q. et al. Structural basis for distinct inflammasome complex assembly by human NLRP1 and CARD8. Nat Commun 12, 188, doi:10.1038/s41467-020-20319-5 (2021).

$62321 \mathrm{Li}$, Y. et al. Cryo-EM structures of ASC and NLRC4 CARD filaments reveal a unified mechanism of nucleation and activation of caspase-1. Proc Natl Acad Sci U S A 115,

62622 Sborgi, L. et al. Structure and assembly of the mouse ASC inflammasome by combined 627 NMR spectroscopy and cryo-electron microscopy. Proc Natl Acad Sci U S A 112, 13237628 13242, doi:10.1073/pnas.1507579112 (2015).

$62923 \mathrm{Lu}$, A. et al. Unified polymerization mechanism for the assembly of ASC-dependent 630 inflammasomes. Cell 156, 1193-1206, doi:10.1016/j.cell.2014.02.008 (2014). 
63124 de Alba, E. Structure and interdomain dynamics of apoptosis-associated speck-like protein 632 containing a CARD (ASC). J Biol Chem 284, 32932-32941, doi:10.1074/jbc.M109.024273 633 (2009).

63425 Nambayan, R. J. T., Sandin, S. I., Quint, D. A., Satyadi, D. M. \& de Alba, E. The 635 inflammasome adapter ASC assembles into filaments with integral participation of its two 636 Death Domains, PYD and CARD. J Biol Chem 294, 439-452,

637 doi:10.1074/jbc.RA118.004407 (2019).

63826 Kuri, P. et al. Dynamics of in vivo ASC speck formation. J Cell Biol 216, 2891-2909, 639 doi:10.1083/jcb.201703103 (2017).

64027 Man, S. M. et al. Inflammasome activation causes dual recruitment of NLRC4 and NLRP3 641 to the same macromolecular complex. Proc Natl Acad Sci U S A 111, 7403-7408, 642 doi:10.1073/pnas.1402911111(2014).

64328 Rigort, A. et al. Focused ion beam micromachining of eukaryotic cells for cryoelectron tomography. Proc Natl Acad Sci U S A 109, 4449-4454, doi:10.1073/pnas.1201333109 (2012).

29 Schaffer, M. et al. Cryo-focused Ion Beam Sample Preparation for Imaging Vitreous Cells by Cryo-electron Tomography. Bio Protoc 5, doi:10.21769/bioprotoc.1575 (2015).

64830 Stutz, A., Horvath, G. L., Monks, B. G. \& Latz, E. ASC speck formation as a readout for 649 inflammasome activation. Methods Mol Biol 1040, 91-101, doi:10.1007/978-1-62703-523650 1_8 (2013)

65131 Smolewski, P. et al. Detection of caspases activation by fluorochrome-labeled inhibitors:

652 Multiparameter analysis by laser scanning cytometry. Cytometry 44, 73-82, 653 doi:10.1002/1097-0320(20010501)44:1<73::aid-cyto1084>3.0.co;2-s (2001).

65432 Wolff, G. et al. Mind the gap: Micro-expansion joints drastically decrease the bending of 655 FIB-milled cryo-lamellae. Journal of Structural Biology 208, 107389, 656 doi:10.1016/j.jsb.2019.09.006 (2019). 
33 Schellenberger, P. et al. High-precision correlative fluorescence and electron cryo microscopy using two independent alignment markers. Ultramicroscopy 143, 41-51, doi:10.1016/j.ultramic.2013.10.011 (2014).

34 Mastronarde, D. N. Automated electron microscope tomography using robust prediction of specimen movements. J Struct Biol 152, 36-51, doi:10.1016/j.jsb.2005.07.007 (2005).

35 Guo, Q. et al. In Situ Structure of Neuronal C9orf72 Poly-GA Aggregates Reveals Proteasome Recruitment. Cell 172, 696-705 e612, doi:10.1016/j.cell.2017.12.030 (2018).

36 Paul-Gilloteaux, P. et al. eC-CLEM: flexible multidimensional registration software for correlative microscopies. Nat Methods 14, 102-103, doi:10.1038/nmeth.4170 (2017).

37 Kremer, J. R., Mastronarde, D. N. \& Mclntosh, J. R. Computer Visualization of ThreeDimensional Image Data Using IMOD. Journal of Structural Biology 116, 71-76, doi:10.1006/jsbi.1996.0013 (1996).

38 Donohoe, B. S. et al. Cis-Golgi cisternal assembly and biosynthetic activation occur sequentially in plants and algae. Traffic 14, 551-567, doi:10.1111/tra.12052 (2013).

39 Hwang, M. S. et al. MAVS polymers smaller than $80 \mathrm{~nm}$ induce mitochondrial membrane remodeling and interferon signaling. The FEBS journal, doi:10.1111/febs.14772 (2019).

40 Ader, N. R. et al. Molecular and topological reorganizations in mitochondrial architecture interplay during Bax-mediated steps of apoptosis. eLife 8, doi:10.7554/eLife.40712 (2019).

$41 \mathrm{Yu}$, J. et al. Inflammasome activation leads to Caspase-1-dependent mitochondrial damage and block of mitophagy. Proc Natl Acad Sci U S A 111, 15514-15519, doi:10.1073/pnas.1414859111 (2014).

42 Schindelin, J. et al. Fiji: an open-source platform for biological-image analysis. Nat Methods 9, 676-682, doi:10.1038/nmeth.2019 (2012).

43 McArthur, K. et al. BAK/BAX macropores facilitate mitochondrial herniation and mtDNA efflux during apoptosis. Science 359, doi:10.1126/science.aao6047 (2018). 
44 Koning, R. I., Koster, A. J. \& Sharp, T. H. Advances in cryo-electron tomography for biology and medicine. Ann Anat 217, 82-96, doi:10.1016/j.aanat.2018.02.004 (2018).

68445 Bauerlein, F. J. B., Fernandez-Busnadiego, R. \& Baumeister, W. Investigating the Structure 685 of Neurotoxic Protein Aggregates Inside Cells. Trends Cell Biol 30, 951-966, doi:10.1016/j.tcb.2020.08.007 (2020).

46 Vyleta, M. L., Wong, J. \& Magun, B. E. Suppression of ribosomal function triggers innate immune signaling through activation of the NLRP3 inflammasome. PLoS One 7, e36044, doi:10.1371/journal.pone.0036044 (2012).

47 Briard, B. et al. Galactosaminogalactan activates the inflammasome to provide host protection. Nature 588, 688-692, doi:10.1038/s41586-020-2996-z (2020).

$48 \mathrm{Xia}, \mathrm{S}$. et al. Gasdermin D pore structure reveals preferential release of mature interleukin1. Nature, doi:10.1038/s41586-021-03478-3 (2021).

49 Mulvihill, E. et al. Mechanism of membrane pore formation by human gasdermin-D. EMBO J 37, doi:10.15252/embj.201798321 (2018).

50 Hagen, W. J. H., Wan, W. \& Briggs, J. A. G. Implementation of a cryo-electron tomography tilt-scheme optimized for high resolution subtomogram averaging. J Struct Biol 197, 191198, doi:10.1016/j.jsb.2016.06.007 (2017).

51 Tegunov, D. \& Cramer, P. Real-time cryo-electron microscopy data preprocessing with Warp. Nat Methods 16, 1146-1152, doi:10.1038/s41592-019-0580-y (2019).

52 Pettersen, E. F. et al. UCSF Chimera--a visualization system for exploratory research and analysis. J Comput Chem 25, 1605-1612 (2004).

53 Martinez-Sanchez, A., Garcia, I., Asano, S., Lucic, V. \& Fernandez, J. J. Robust membrane detection based on tensor voting for electron tomography. J Struct Biol 186, 49-61, doi:10.1016/j.jsb.2014.02.015 (2014). 
54 Ader, N. R. \& Kukulski, W. triCLEM: combining high-precision, room temperature CLEM with cryo-fluorescence microscopy to identify very rare events. Method Cell Biol 140, 303320, doi:10.1016/bs.mcb.2017.03.009 (2017).

55 Kukulski, W. et al. Correlated fluorescence and 3D electron microscopy with high sensitivity and spatial precision. Journal of Cell Biology 192, 111-119, doi:10.1083/jcb.201009037 (2011).

\section{Acknowledgements}

714 We acknowledge the MRC Laboratory of Molecular Biology (LMB) Electron Microscopy (EM)

715 Facility for access and support in EM sample preparation and data collection, and the Flow

716 Cytometry Facility for access and training. We are grateful to Dustin Morado for assisting in

717 cryo-ET data collection and image processing. We acknowledge the MRC LMB Light

718 Microscopy Facility, in particular Jonathan Howe, for access and support imaging data

719 collection. We are grateful for access to the MRC-LMB Scientific Computing facilities. We thank

720 Kendra E. Leigh, Wanda Kukulski, Emma Jones, Robert Pickering, Maria Daly, Victoria L. Hale

721 and Zunlong Ke for helpful advice and discussions. This work was supported by Wellcome

722 Senior Research Fellowship 217191/Z/19/Z to Y.M.; a PhD studentship to Y.L. from the China

723 Scholarship Council and Cambridge Trust; and Wellcome Investigator Award 108045/Z/15/Z to

724 C.E.B.

\section{Author contributions}

726 Y.L., L.J.H., C.E.B and Y.M. conceived the experiments. Y.L., H.Z. and J.H. collected light

727 microscopy data. Y.L. and J.B. analysed light microscopy data. Y.L., A.C.B. and C.H. prepared 728 cryo-EM samples, performed CLEM imaging and collected cryo-ET data. Y.L. processed the 729 cryo-ET data. Y.L. and H.A. analysed the cryo-ET data and flow-cytometry analysis. Y.L. and 
730 L.J.H. performed cell-based assays. Y.L, C.E.B and Y.M. wrote the manuscript with input from

731 all authors. Project supervision, administration and funding acquisition, C.E.B. and Y.M.

\section{Competing interests}

733 C.E.B are Y.M. are consultants for Related Sciences LLC and have profits interests in Danger

734 Bio LLC. CEB is on the SAB of NodThera and Lightcast.

735

736 
740

741

742

743

744

745

746

747

748

\author{
Liu et al. \\ Supplementary Information \\ Supplementary Materials and Methods \\ Figures S1-S5
}




\section{Supplementary Materials and Methods}

750 Correlative fluorescence microscopy and electron tomography of resin-embedded cells.

751 Correlative fluorescence microscopy and electron tomography (RT-CLEM) of resin-embedded

752 cells was performed as described ${ }^{40,54}$. Briefly, ASC-mCerulean cells were grown on carbon-

753 coated $3 \mathrm{~mm}$ sapphire discs (Wohlwend $\mathrm{GmbH}$ ) in two-well chambers (ibidi) for $24 \mathrm{~h}$ before

754 priming and NLRPC-driven inflammasome stimulation as described above. Cells were stained

755 with MitoView far-red and FLICA before high pressure freezing with an HPM100 high pressure

756 freezing system (Leica Microsystems). Grids were imaged with a cryo-fluorescence microscope

757 (Leica EM Cryo CLEM, Leica Microsystems) to localise cells containing ASC-mCerulean

758 specks. Freeze substitution was preformed using $0.008 \%$ uranyl acetate in acetone and

759 embedded in Lowicryl HM20 (Polysciences) using an AFS2 (Leica Microsystems). Blocks were

760 sectioned into $300 \mathrm{~nm}$ thin sections using a microtome (Leica Microsystems) equipped with a

761 diamond knife (Diatome). The sections were collected on 200 mesh/300 mesh copper grids with

762 carbon support (Agar Scientific Ltd). TetraSpeck 100-nm microspheres were diluted 1:100 in

763 PBS and applied to the sections for use as fiducial markers for correlation. Sections were

764 imaged on a Nikon Ti2 wide field microscope equipped with a Niji LED light source (Bluebox

765 Optics, Blackwood, UK), a x100/1.49 NA Apo TIRF oil immersion objective lens and a Neo

766 sCMOS DC-152Q-C00-FI camera (Andor Technology, Belfast, UK). Filters: mCerulean (89006

767 filter set, Chroma Technology, Bellows Falls, USA), fluorescein (49002 filter set, Chroma),

768 MitoView Far Red (49006 filter set, Chroma). EM images were collected using a Tecnai F20

769 electron microscope (ThermoFisher) operated at $200 \mathrm{kV}$ and a high-tilt tomography holder

770 (Fischione Instruments; Model 2020). An image montage of regions of interest was acquired on

771 a BM-Orius detector using TEM mode at 150-200 $\mu \mathrm{m}$ defocus using SerialEM v3.8.0 $0^{34}$ at a pixel

772 size of $1.1 \mathrm{~nm}$. The correlation between the montaged map and fluorescent map was performed

773 by image transformation of registered fiducial markers in both image modalities as previously 
bioRxiv preprint doi: https://doi.org/10.1101/2021.09.20.461078; this version posted September 20, 2021. The copyright holder for this preprint (which was not certified by peer review) is the author/funder. All rights reserved. No reuse allowed without permission.

774 described ${ }^{55}$. Tilt-series were acquired from approximately -60 to +60 with $1^{\circ}$ increment at a pixel

775 size of $1.1 \mathrm{~nm}$. Samples were rotated $90^{\circ}$ to acquire dual axis tilt-series. Tomograms were

776 reconstructed and visualised with $\mathrm{IMOD}^{37}$.

779

780 

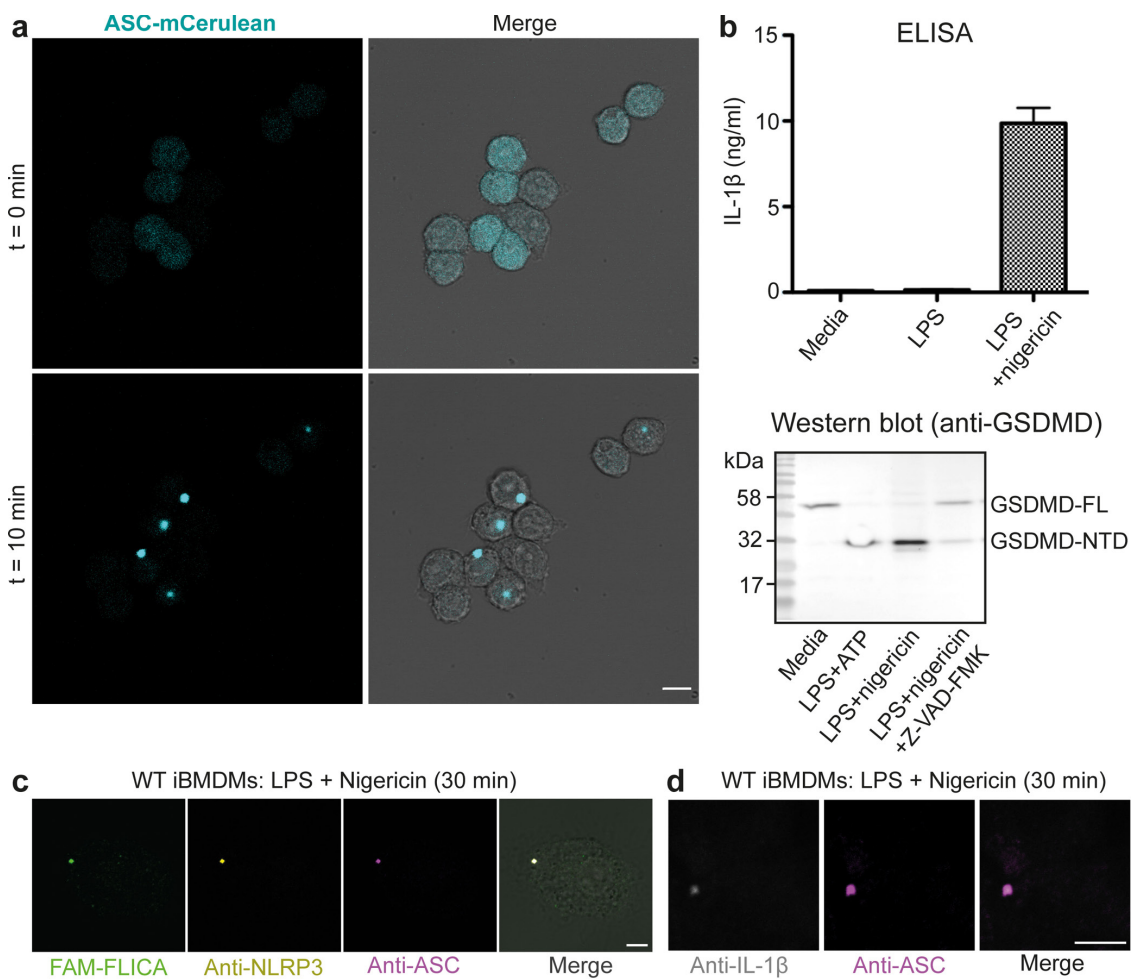

d WT iBMDMs: LPS + Nigericin (30 min)

Figure S1. Tracking NLRP3 inflammasome activation in iBMDMs with fluorescence microscopy and biochemical assays.

(a) Snapshots of live iBMDMs expressing ASC-mCerulean. ASC specks formed within 10 min of NLRP3 inflammasome activation with LPS and nigericin. Merge, overlay of fluorescence and bright-field images. Scale bar, $10 \mu \mathrm{m}$.

(b) Biochemical assays of inflammasome activation in ASC-mCerulean iBMDMs: upper panel, ELISA measuring IL-1 $\beta$ secretion; lower panel, Western blot showing proteolytic cleavage of GSDMD following different stimuli. FL, full-length. NTD, N-terminal domain.

(c) Fluorescence microscopy of WT iBMDMs stimulated with LPS and either nigericin or ATP. Caspase-1 was detected with FAM-FLICA. NLRP3 and ASC were detected by immunostaining. Scale bars, $5 \mu \mathrm{m}$.

(d) Immunofluorescence microscopy of WT iBMDMs stimulated with LPS and either nigericin or ATP, with staining for IL-1 $\beta$ and ASC. Scale bars, $5 \mu \mathrm{m}$.

(e) Snapshots of live ASC-mCerulean iBMDMs stained with FAM-FLICA after nigericin stimulation showing ASC-mCerulean colocalization with caspase-1 and FAM-FLICA fluorescence intensity increasing over time. Scale bar, $5 \mu \mathrm{m}$. 
a

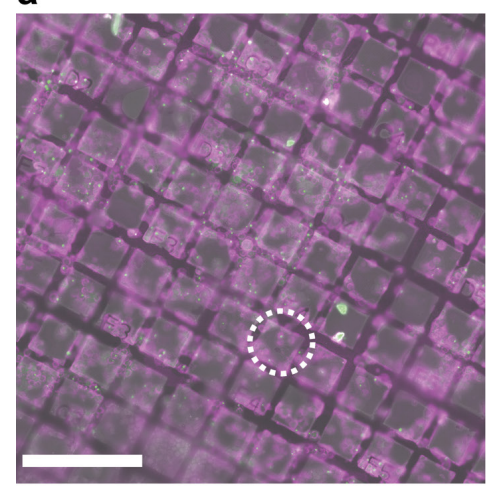

d

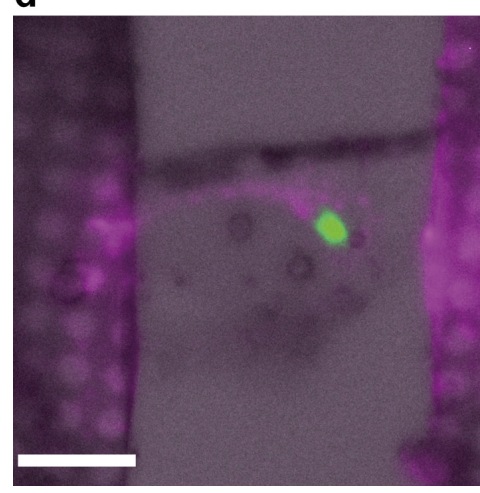

b

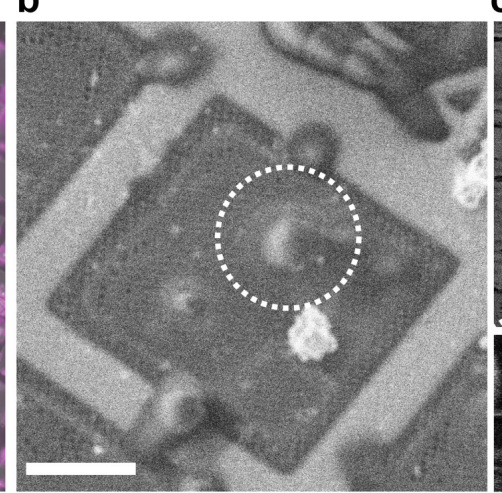

e

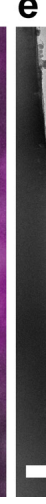

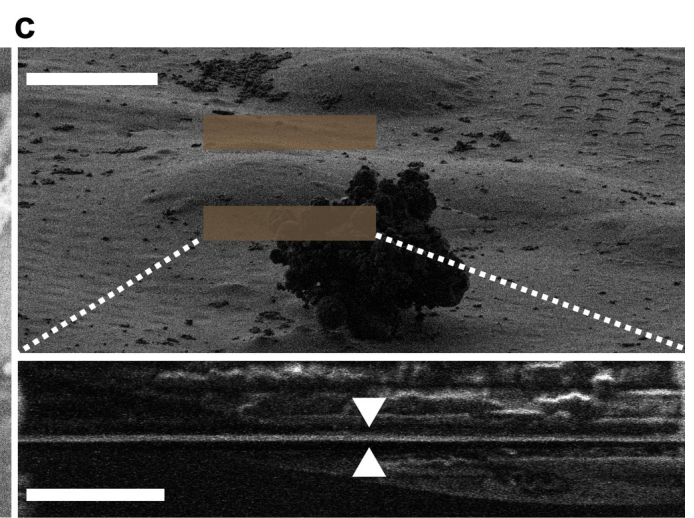

f

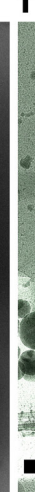

g

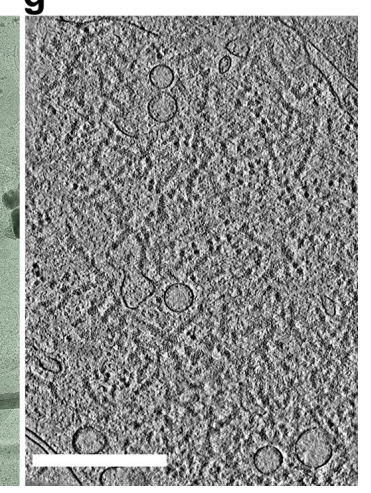

799 Figure S2. Workflow for correlative cryo-light and electron microscopy (cryo-CLEM).

800 (a) Cryo-fluorescence microscopy (cryo-FM) of ASC-mCerulean iBMDMs, with brightfield image

801

802

803

804

805

806 overlaid. Cells were cultured on finder grids, primed with LPS, stimulated with nigericin for $30 \mathrm{~min}$, labelled with MitoView and FAM-FLICA, vitrified, and then imaged. The white circle denotes one area of interest. Scale bar: $300 \mu \mathrm{m}$.

(b) Grids were transferred to a scanning electron microscope (SEM) fitted with a focused-ionbeam (FIB). The cell containing the area of interest, identified using the finder-grid markers, was imaged by SEM. Scale bar, $40 \mu \mathrm{m}$.

(c) SEM images of the area of interest before and after FIB milling: Upper panel, before FIB milling with initial target milling windows shown in brown (scale bar, $10 \mu \mathrm{m}$ ); lower panel, side-on view of the lamella after FIB milling (scale bar, $4 \mu \mathrm{m}$ ).

(d) Cryo-FM image of a lamella containing an ASC speck with brightfield image overlaid. Scale bar, $10 \mu \mathrm{m}$.

(e) Low magnification transmission cryo-EM map of the lamella shown in (d). Scale bar, $7 \mu \mathrm{m}$.

(f) Medium magnification cryo-EM map overlaid with the cryo-FM map after registration and transformation. The region of interest for tilt-series acquisition is boxed. Scale bar, $1 \mu \mathrm{m}$.

(g) Reconstructed tomographic slice acquired in the area marked in (f) containing the fluorescent signal. Several short filaments and small trans-Golgi-like vesicles were visible. Scale bar, $300 \mathrm{~nm}$. See Movie S4 for a tomogram of this region. 
a

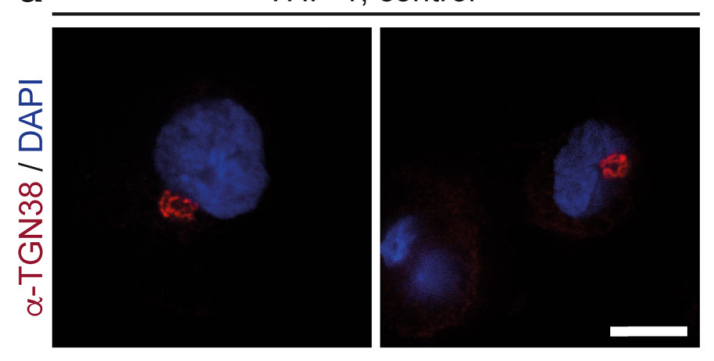

THP-1, LPS + nigericin (30 min)

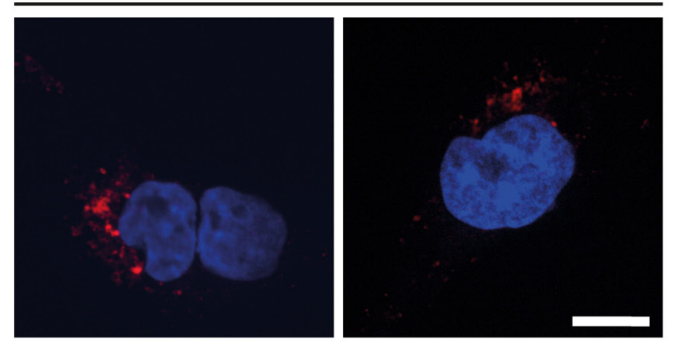

b
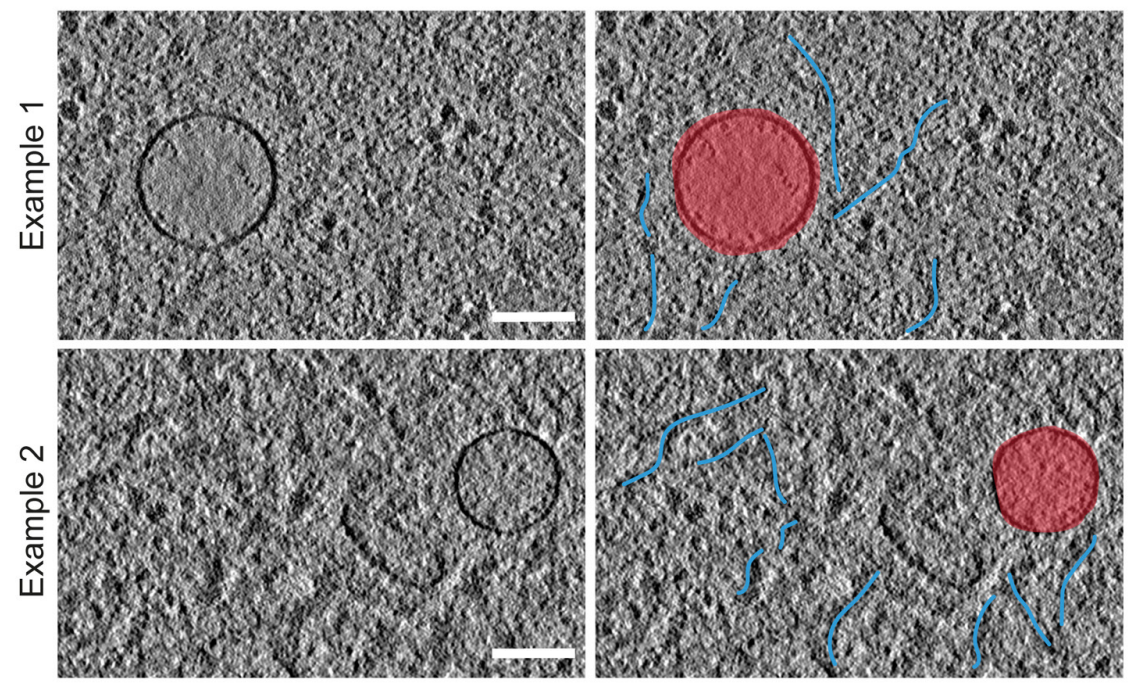

C

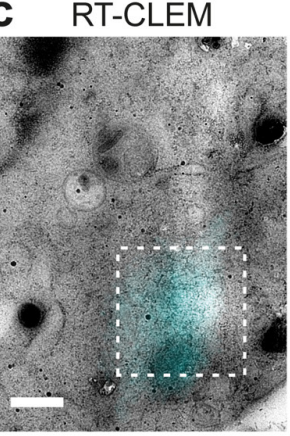

ASC-mCerluean

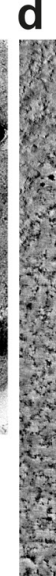

e RT-CLEM vesicles in puncta
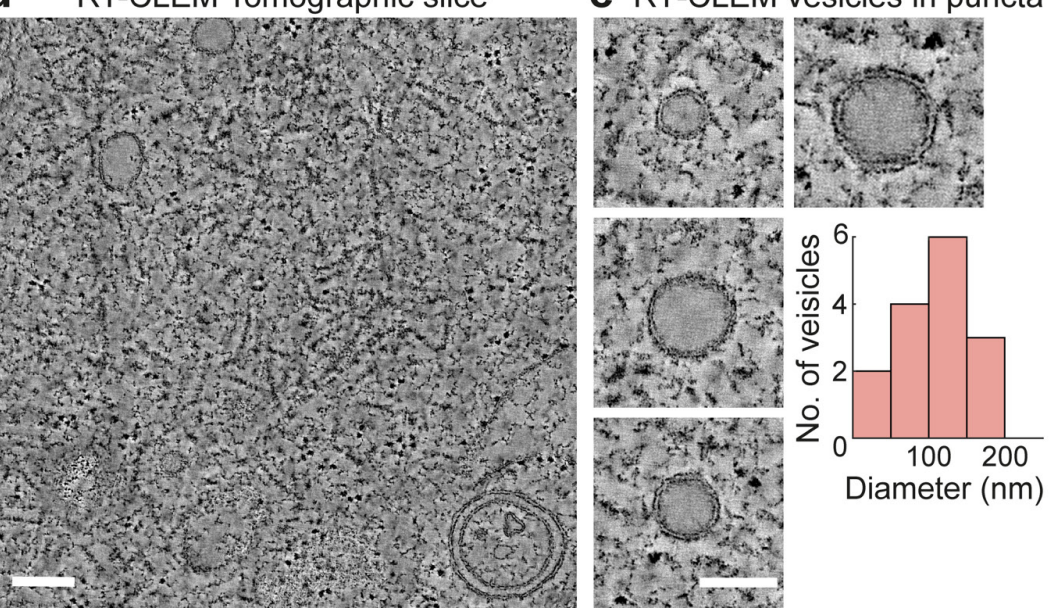

\section{Figure S3. Imaging of trans-Golgi-like vesicles within ASC puncta.}

(a) Fluorescence micrographs of THP-1 cells with and without stimulation with LPS and nigericin, with TGN (anti-TGN38) immunostaining. Scale bar, $10 \mu \mathrm{m}$.

(b) Two tomographic slices of ASC-mCerulean speck showing vesicles adjacent to filaments. Scale bars, $100 \mathrm{~nm}$.

(c-e) CLEM of ASC-mCerulean iBMDMs stimulated with nigericin for $30 \mathrm{~min}$, high-pressure frozen, chemically fixed, sectioned and imaged at room temperature (see Supplementary Materials and Methods). (c) Low magnification EM map overlaid with ASC-mCerulean fluorescence. Scale bar, $1 \mu \mathrm{m}$. (d) $5.5 \mathrm{~nm}$-thick tomographic slice of in the region boxed in (c). Scale bar, $200 \mathrm{~nm}$. (e) Tomographic slices showing vesicles with low-density lumens within ASC-mCerulean puncta. Scale bar, $100 \mathrm{~nm}$. The histogram shows the vesicle diameter distribution. 


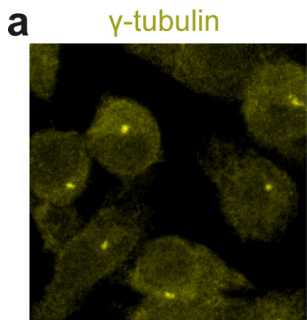

Pericentrin

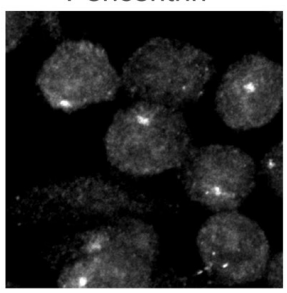

ASC-mCerulean

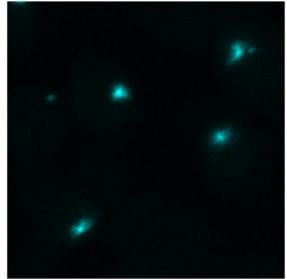

ASC-mCerulean

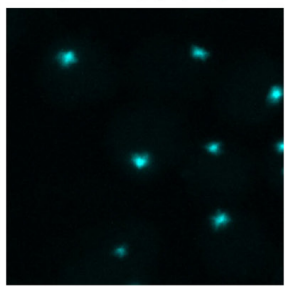

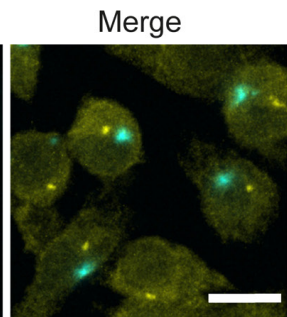

Merge

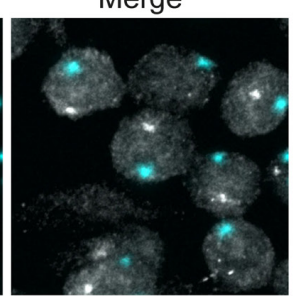

Measured distance distribution

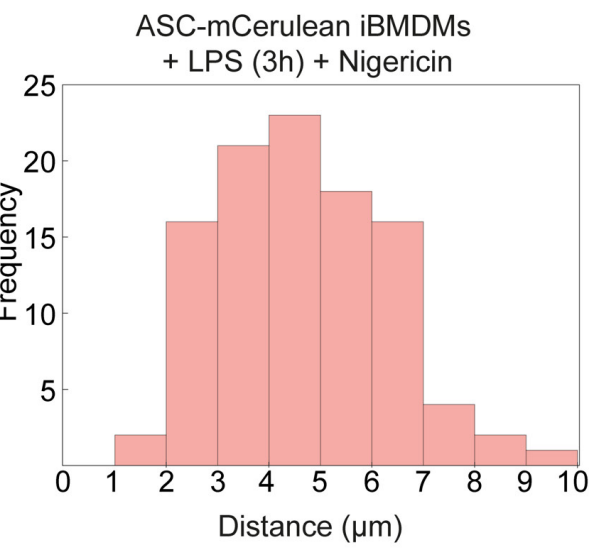

b iBMDMs + LPS (3h priming) +Nigericin (30 mins)

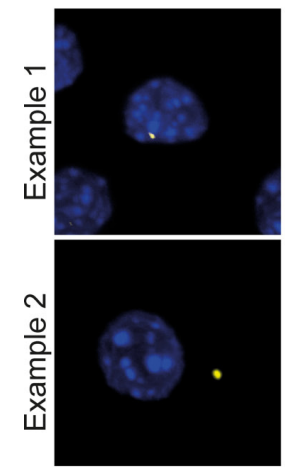

Y-tubulin
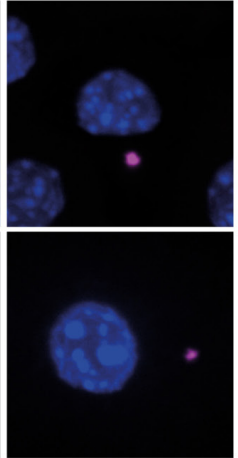

ASC

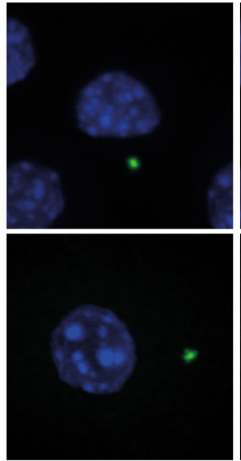

FAM-FLICA

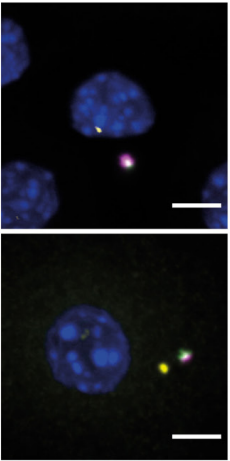

Merge

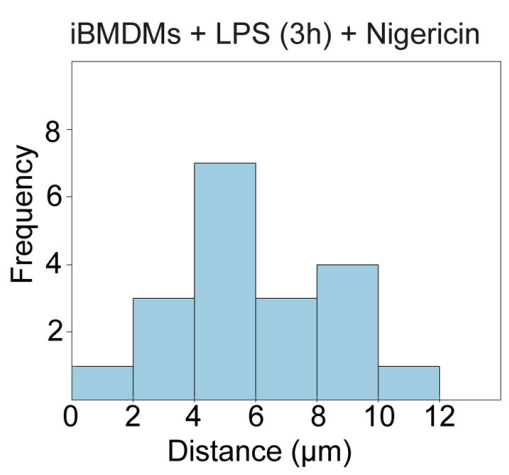

C

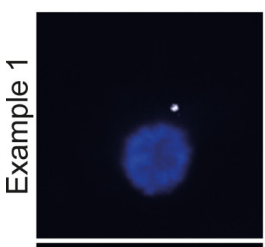

THP-1 + LPS (3h priming) +Nigericin (30 mins)

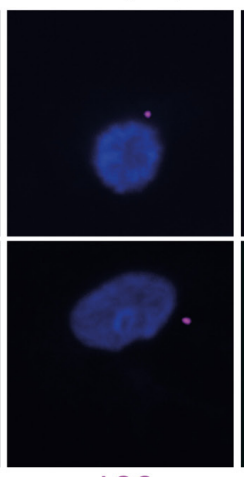

ASC

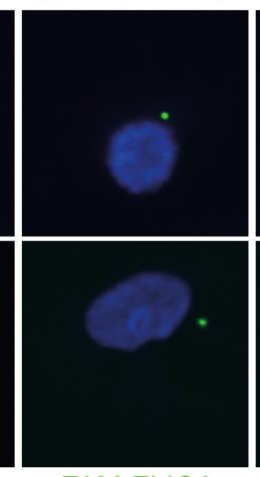

FAM-FLICA

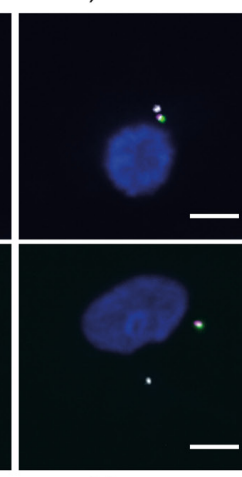

Merge

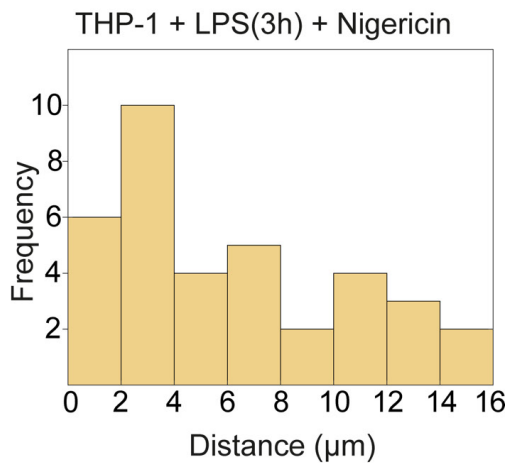

Figure S4. Localization of ASC and caspase-1 relative to MTOC-associated components.

(a) Fluorescence micrographs of ASC-mCerulean iBMDMs stimulated with LPS and nigericin, with $\gamma$-tubulin immunostaining. Scale bar, $10 \mu \mathrm{m}$. Right, distribution of the distance between ASC-mCerulean and anti- $\gamma$-tubulin fluorescence.

(b) Fluorescence micrographs of WT iBMDMs stimulated with LPS and nigericin. Cells were stained with FAM-FLICA, and with antibodies against $\gamma$-tubulin and ASC. Scale bar, $10 \mu \mathrm{m}$. Right, distribution of the distance between ASC/FAM-FLICA and anti- $\gamma$-tubulin fluorescence.

(c) Fluorescence micrographs of THP-1 cells stimulated with LPS and nigericin. Cells were stained with FAM-FLICA, and with antibodies against ninein and ASC. Scale bar, $10 \mu \mathrm{m}$. Right, distribution of the distance between ASC/FAM-FLICA and anti-ninein fluorescence. 

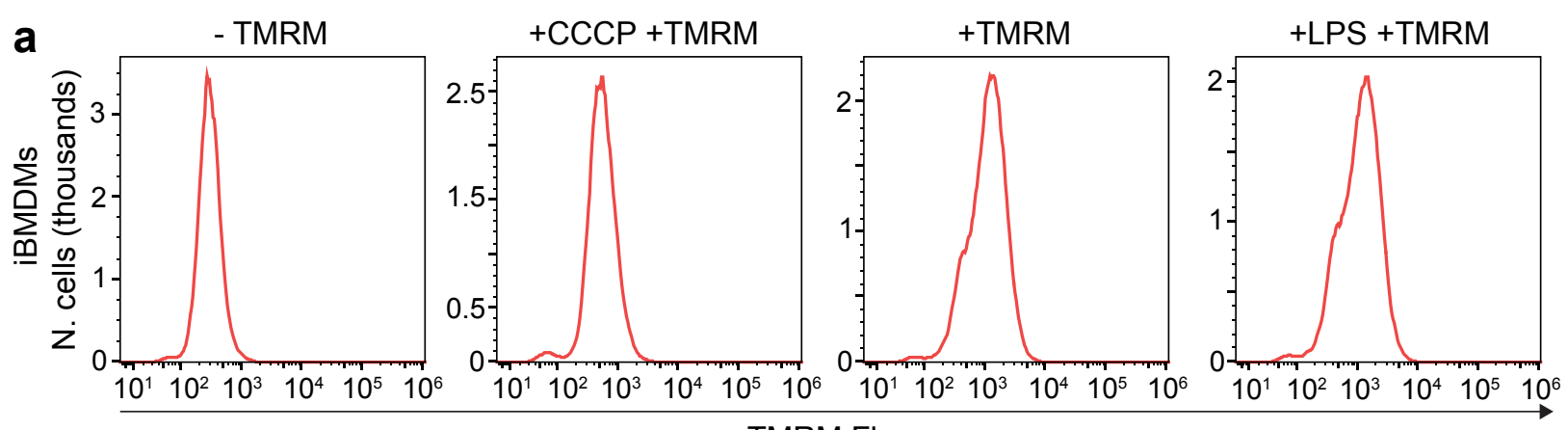

TMRM Fluorescence

b iBMDM type

Nigericin stimulation ( $\mathrm{min}$ )

Gap width (nm)

Estimated gap depth

\begin{tabular}{c}
\hline ASC-mCerulean \\
ASC-mCerulean \\
ASC-mCerulean \\
ASC-mCerulean \\
WT \\
WT
\end{tabular}

30

30

30

60

60

11
15
18
11
20
17
(nm)

11

12

14

11

20

$-$

17

17

Figure S5. Functional and ultrastructural analyses of mitochondria in iBMDMs stimulated with nigericin.

(a) Additional flow cytometry histograms of iBMDMs. The following controls are shown to accompany Fig. 4b: cells with no TMRM staining; cells stained with TMRM and a potent mitochondrial uncoupling chemical, carbonyl cyanide 3- chlorophenylhydrazone (CCCP); cells stained with TMRM only; and cells primed with LPS and stained with TMRM.

(b) Measured sizes of outer-membrane discontinuities in cryo-ET reconstructions of mitochondria in WT and ASC-mCerulean iBMDMs, as partly shown in Fig. 5c-e. 